\title{
Sequence Comparison of Wild-Type and Cold-Adapted B/Ann Arbor/1/66 Influenza Virus Genes
}

\author{
DAN C. DEBORDE, ${ }^{\prime}$ ARMEN M. DONABEDIAN, M. LOUISE HERLOCHER, \\ CLAYTON W. NAEVE, ${ }^{*}$ AND HUNEIN F. MAASSAB ${ }^{2}$
}

Department of Epidemiology, School of Public Health, University of Michigan, 109 Observatory St., Ann Arbor, Michigan, 48109, and
* Elepartment of Virology and Molecular Biology, St. Jude Children's Research Hospital, Memphis, Tennesse日, 38101

Received August 10, 1987; accepted November 24, 1987

\begin{abstract}
Consensus sequences for both wt and ca B/Ann Arbor/1/66 viral PB2, PB1, PA, NP, M, and NS genes were directly determined from vRNA using a combination of chemical and chain-termination sequencing methods. There were 105 sites of difference between the wt and ca sets of these six RNA genes. The differences resulted in 26 amino acid substitutions distributed over the six proteins. The sequence changes were compared to the sequences of other known influenza type $B$ wt viruses to pinpoint those changes that were unique to the ca B/Ann Arbor/1/66 virus. Of the 26 amino acid differences, only 11 were unique to the cold-adapted virus. These unique sites were distributed among five of the six genes. The NS protein had no amino acid substitutions. The sequence changes are discussed in terms of their probable mode of origin and selection, and in terms of their importance to the cold-adapted, temperature-sensitive, and attenuation phenotypes of $\mathrm{ca} B / \mathrm{AA} / 1 / 66$ virus. The sequence and organization of the $\mathrm{PB} 2 \mathrm{gene}$ and predicted protein are also given. The PB2 gene was 2396 nucleotides long, and it encoded a predicted protein of 770 amino acids with a molecular weight of $88,035 \mathrm{Da}$ for the wt virus and 88,072 Da for the ca virus. Both proteins were predominantly hydrophilic, and each had an overall charge of +24.5 at $\mathrm{pH} 7.0$. (c) 1988 Academic Press, Inc.
\end{abstract}

\section{INTRODUCTION}

The cold-adapted (ca) B/Ann Arbor/1/66 (B/AA/1/66) influenza virus line was recently chosen for use in the production of ca reassortant live virus vaccines for testing in man (Maassab et al., 1985). The rationale behind the production of these ca reassortant vaccines is based on the experience amassed from successful triats using influenza type $A$ ca reassortant vaccines. The optimal vaccine reassortant derives the viral genes encoding the two surface antigens, the hemagglutinin (HA) and the neuraminidase (NA), from the wild-type (wt) parent. Its other six genes are derived from the ca donor virus, and it is these genes which carry the characteristic ca, temperature-sensitive (ts), and attenuated (att) phenotypes. Thus, for the purposes of a safe and effective influenza $A$ virus vaccine, all that is required is the assured presence of all six nonsurface genes from the ca A/Ann Arbor/60 donor virus (for review, Maassab et al., 1985). Laboratory studies have confirmed this phenotypic transfer to influenza $B$ reassortant viruses by the six nonsurface genes of ca $B / A A 1 / 66$ in primary chick kidney (PCK) tissue culture and in ferrets (Maassab et al., 1986).

\footnotetext{
'Present address: Department of Microbiology, University of Montana, Missoula, MT 59812.

${ }^{2}$ To whom requests for reprints should be addressed.
}

Human trials have been done and are being done to extend the validity of this approach for ca influenza B reassortant vaccine viruses (Davenport et al., 1977; Monto et al., 1982; Reeve et al., 1982; Keitel et al., 1986). While the transfer of these phenotypes is guaranteed by the transfer of all six nonsurface genes, the total number of mutations which brings about the ca, ts, and att phenotypes and their locations in the genome are not known. It is also not clear whether these phenotypes are engendered only by the mutations that were selected by the cold-adaptation process, or by other mutations selected in an unknown manner.

Initial studies used various methods to reveal that each of the eight genes of the ca AvAnn Arbor/6/60 (A/AA/6/60) donor virus displayed some difference from the corresponding gene of the wt $A / A A / 6 / 60$ virus from which it was derived (Cox et al., 1986). Such genomic-wide changes are also probable with the influenza $B$ virus system since just the electrophoretic comparison of the wt and $c a B / A A 1 / 66$ viral RNA segments demonstrated that changes occur in at least six of the eight genes (Maassab et al., 1985). Only the NA and NS ca and wt gene pairs did not migrate differently under the various conditions tested. Other studies for both influenza $A$ and $B$ viruses (Snyder et al., 1987; Donabedian et al., 1987) have used a genetic approach in an attempt to analyze the particular gene(s) associated with the three phenotypes. They 
isolated reassortants containing all wt influenza genes except for one gene from the ca parent virus. These "single-gene" reassortants were then analyzed for expression of the ca, ts, and att phenotypes. Singlegene analysis works reasonably well for the ts and att phenotypes, but less well for the ca phenotype since the ability to replicate efficiently at $25^{\circ}$, not a normal wt virus characteristic, can be inhibited by the presence of any wt gene that does not function well at this suboptimal temperature. In addition, some ca viral genes may act only in concert with one or more of the other ca donor virus genes to express these phenotypes, and hence, not be identified by single-gene studies.

In an effort to determine the total extent of the sequence changes that were selected in the six nonsurface genes during the cold-adaptation of $B / A A 1 / 66 w t$ virus, we sequenced both the wt and $\mathrm{ca} B / A \mathrm{~A} / 1 / 66$ strains' PB2, PB1, PA, NP, M, and NS genes. These sequence data provide a complete catalog of the nucleotide changes that existed between these two virus lines, and these changed sites were compared to other known wt influenza $B$ virus sequences to pinpoint changes unique to $\mathrm{ca} B / \mathrm{AA} / / 66$ viral genes. These data coupled with information derived from the single-gene reassortment studies mentioned above will provide a base of knowledge for future experiments which will attempt to link particular mutations to specific phenotypes. (See following article, Donabedian et al., 1988).

\section{MATERIALS AND METHODS}

\section{Chemicals and enzymes}

Oligodeoxynucleotide primers were purchased from the University of Michigan DNA Synthesis Facility or were made by one of the authors, C. W. Naeve, with an Applied Biosystems Model 380A DNA Synthesizer. The deoxynucleoside triphosphates, dimethyldichlorosilane, $\gamma$-(methacryloxy)propyltrimethoxysilane, diethyl pyrocarbonate (DEP), and sodium borohydride were purchased from Sigma Chemical Co., St. Louis, Missouri. The dideoxynucleoside 5'-triphosphates were obtained from Boehringer-Mannheim, Indianapolis, Indiana. Reverse transcriptase was the standard preparation derived from avian myeloblastosis virus by Life Sciences, Inc., St. Petersburg, Florida. Terminal deoxynucleotidyl transferase (TdT) was either from Life Sciences, Inc., or from Bethesda Research Laboratories, Gaithersburg, Maryland. T4 RNA ligase and T4 polynucleotide kinase were also from Bethesda Research Laboratories. Dimethyl sulfate and aniline were from Aldrich Chemical Co., Milwaukee, Wisconsin. Anhydrous hydrazine was purchased from Fisher Sci- entific Co., Fair Lawn, New Jersey. [ $\left.\alpha^{32} \mathrm{P}\right]$ deoxyadenosine $5^{\prime}$-triphosphate, tetra-(triethylammonium) salt was purchased from Dupont, NEN Research Products, Boston, Massachusetts, while $\left[\gamma^{32}\right.$ P]adenosine $5^{\prime}$-triphosphate was from ICN Radiochemicals, Irvine, California.

\section{Viruses and tissue culture}

The viruses studied in these experiments were wt and $\mathrm{ca} B / A \mathrm{~A} 1 / 66$ strains, and wt B/Melbourne/43 and $B / A A / 1 / 86$ viruses. The cold-adaptation of the wt $\mathrm{B} / \mathrm{AA} / 1 / 66$ virus together with the passage histories for both wt and ca viruses are given in Fig. 1. The original isolate was cultured in primary chick kidney cells from a human nasal wash sample (Maassab et al., 1986). Il was not cloned, and therefore the starting virus was a population of individual viruses probably of related but varying individual sequences. The production of viral stocks for the isolation of VRNA for sequencing was performed exactly as presented previously (DeBorde et al., 1986).

\section{Preparation of vRNA}

The isolation of $V R N A$ was performed as presented previously (DeBorde et al., 1986), except that the RNA from virus grown in 60-100 embryonated eggs was

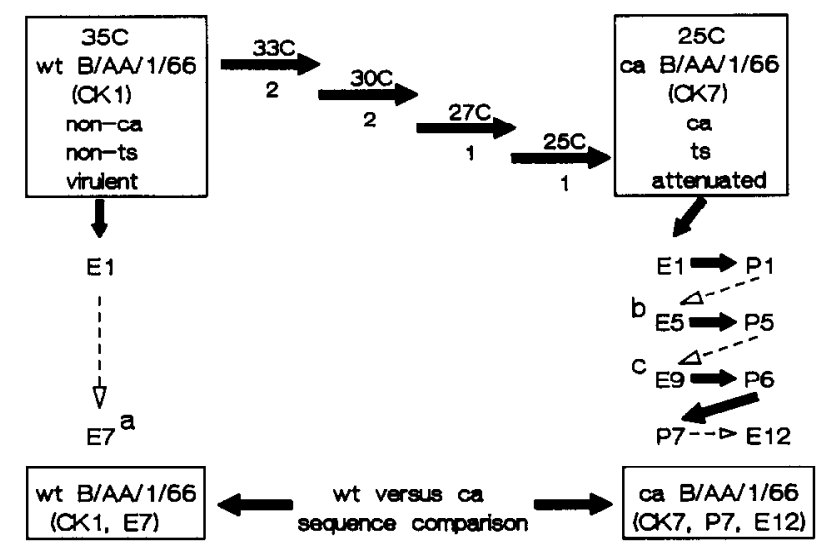

FIG. 1. Cold-adaptation and passage history of wt and ca B/AN/1/ 66 viruses. The cold-adaptation process is represented by the staggered arrows connecting the top two boxes. The passages were done in PCK cells at the temperatures indicated above each arrow. The number below the arrow represents number of passages at each temperature. The dashed arrows represent a number of replicative steps as follows: ${ }^{\circ}$ Consecutive embryonated egg passages of the wt virus pool at high dilution. ${ }^{\circ}$ Consecutive plaquing and egg amplification of the ca virus pool. "Consecutive embryonated egg passages of the ca virus pool at high dilution. The bottom two boxes represent the $w t$ and $\mathrm{ca}$ virus pools that were sequenced in this paper. 
resuspended in a final volume of $50 \mu \mathrm{l}$, not $50 \mathrm{ml}$ as erroneously stated in that paper.

\section{End-labeling of vRNA}

Purified vRNA (30 to $50 \mu \mathrm{g}$ ) and $100 \mu \mathrm{Ci}$ of [ [32P]cytidine bis-phosphate $(p C p)$ were dried down together in a vacuum concentrator and resuspended in $5 \mu$ of $2 X$ RNA ligase buffer, (100 mM HEPES, pH 7.5, $20 \mu \mathrm{g} / \mathrm{ml}$ BSA, $6.6 \mu M$ ATP, $\left.30 \mathrm{mM} \mathrm{MgCl})_{2}\right), 1 \mu$ l dimethylsulfoxide, and $1 \mu l 0.5 \mathrm{mM}$ ATP. Thirty to thirty-five $\mathrm{pCp}$ ligation unis of T4 RNA ligase was added, and the total volume was adjusted to $10 \mu \mathrm{l}$ with $\mathrm{H}_{2} \mathrm{O}$ if necessary. The reaction was incubated at $4^{\circ}$ overnight. Two hundred microliters of $0.3 \mathrm{M}$ sodium acetate was added to the ligation mixture, followed by $600 \mu \mathrm{l}$ of 95\% ethanol, and the RNA was precipitated at $-20^{\circ}$ for at least $4 \mathrm{hr}$. The RNA was pelleted for $5 \mathrm{~min}$ in a microfuge, washed once with $70 \%$ ice-cold ethanol, pelleted once more, and dried in a vacuum concentrator. The RNA was resuspended in $\mathrm{H}_{2} \mathrm{O}$ and mixed with an equal volume of loading buffer (0.2X TBE, $0.2 \%$ SDS, $20 \%$ sucrose, and $0.01 \%$ bromphenol blue).

\section{Isolation of individual VRNA segments}

For the chemical sequencing reactions, the purified and $\left.{ }^{32} \mathrm{P}\right] \mathrm{PCP} 3^{\prime}$ end-labeled VRNA was separated into its eight segments on a $3 \%$ polyacrylamide gel in $1 \times$ TBE, (0.09 $M$ Trizma base, $0.09 \mathrm{M}$ boric acid, and 2 $m M \mathrm{Na}_{2} \mathrm{EDTA}$ ) (Peacock and Dingman, 1968) with $0.01 \%$ sodium dodecyl sulfate (SDS). This level of SDS still protected the RNA from any contaminating RNases during the long electrophoretic run, but did not preclude visualization of the RNA with ethidium bromide. These conditions separated all segments except RNAs 4 and 5 (NP and HA). To separate these two RNAs, $6 \mathrm{M}$ urea was added to the above gel. The RNA was electrophoresed at $37^{\circ}$ for $16 \mathrm{hr}$ at $200 \mathrm{~V}$, constant voltage for the nondenaturing gel, and at $37^{\circ}$ for $21 \mathrm{hr}$ at $240 \mathrm{~V}$, constant voltage for the $6 \mathrm{M}$ urea gel. After electrophoresis the vRNA scgments were visualized by immersing the gel in 1 liter of $1 X$ TBE buffer containing $0.01 \%$ SDS and $1 \mu \mathrm{g} / \mathrm{ml}$ ethidium bromide for $25 \mathrm{~min}$ at room temperature. The gel was then removed from the ethidium bromide solution, rinsed with $1 \times \mathrm{TBE}$ buffer, and placed on a sheet of plastic wrap on a uv transilluminator. The desired RNA was excised from the gel with little or no excess gel material. The end-labeled VRNA segments were then eluted from the gel slices using an International Biotechnologies, Inc. (New Haven, CT), unidirectional electroeluter. The running buffer was $0.5 \times$ TBE buffer plus $0.01 \%$ sarcosine. The high salt re- tention buffer was $3.0 \mathrm{M}$ sodium acetate with $0.03 \%$ sarcosine and $0.01 \%$ bromphenol blue. Elution proceeded at $100 \mathrm{~V}$, constant voltage, for l-hr intervals. The smaller segments, NS and $M$, were completely eluted in $1 \mathrm{hr}$; NP, HA, and NA segments took 2 to 3 $\mathrm{hr}$; and the three polymerase segments, PB2, PB1 and PA took 3 to $4 \mathrm{hr}$. Completion of elution was monitored by Cerenkov counting of the original gel slice and hourly samples. Recovery was 70 to $90 \%$ of the original sample. The isolated VRNA segments were precipitated at $-20^{\circ}$ overnight with 3 vol of $95 \%$ ethanol.

\section{Chain-termination sequencing reactions and gels}

These procedures, including the use of TdT to eliminate ambiguities, were performed exactly as described previously (DeBorde et al., 1986). Only one compression area was encountered. It was in the PB2 gene and was resolved by running the standard sequence reaction products on a gel that was a $7 \mathrm{M}$ urea, $8 \%$ polyacrylamide gel modified by the addition of $40 \%$ deionized formamide (Martin, 1987). To determine the wt and ca sequences, the sequencing reaction products were run side by side on the same electrophoretic gel. Several independent runs, with and without TdT enzyme treatment, were performed for each primer.

\section{Chemical sequencing reactions and gels}

These procedures were taken from Peattie (1979) and followed exactly, including the electrophoresis and autoradiography procedures. Recommendations given in Peattie (1979) for the safe use and disposal of sodium borohydride, aniline, DEP, and hydrazine were followed.

\section{Sequence analysis programs}

All sequence analyses, comparisons, manipulations, and calculations were performed using the programs developed by Queen and Korn (1984) and distributed by Beckman Instruments, Inc., Palo Alto, California, as the Microgenie Sequence Software package.

\section{RESULTS}

Figures 2-7 present the cRNA (plus strand) sequences and the predicted polypeptide sequences for the PB2, PB1, PA, NP, M, and NS genes. Each gene sequence was determined by a combination of chemical and chain-termination sequencing procedures (see Materials and Methods for details and/or 


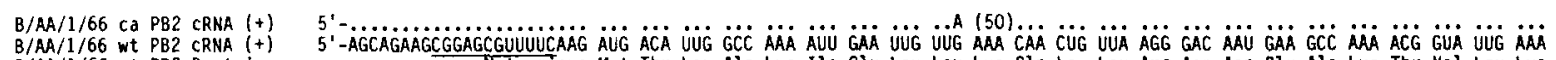

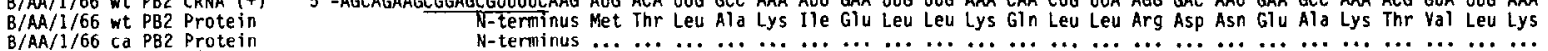

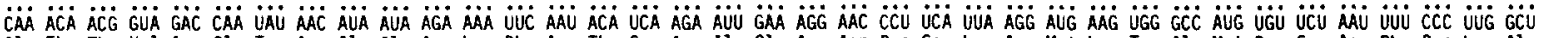
Gln Thr Thr yal Asp Gln Tyr Asn 11 le Ile Arg Lys Phe Asn Thr Ser Arg Ile Glu Arg Asn Pro Ser Leu Arg Met Lys Trp Ala Met Cys Ser Asn Phe Pro Leu Ala

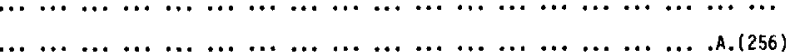

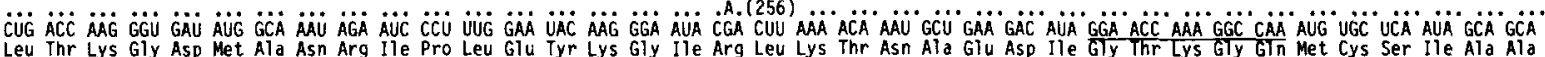

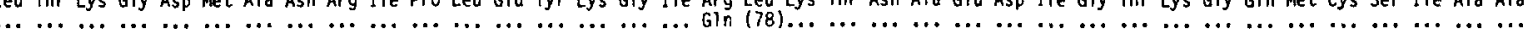

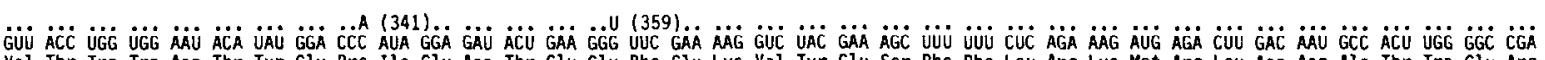

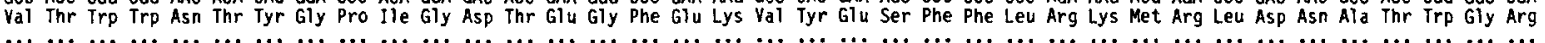

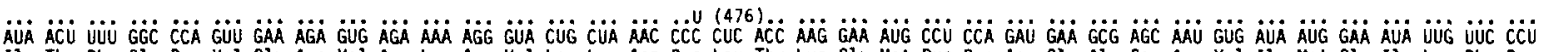
Ile Thr Phe Gly Pro val Glu Arg val Arg Lys Arg val Leu Leu Asn Pro Leu Thr Lys glu Met Pro Pro Asp glu Ala Ser Asn val tle Met glu Ile Leu Phe Pro

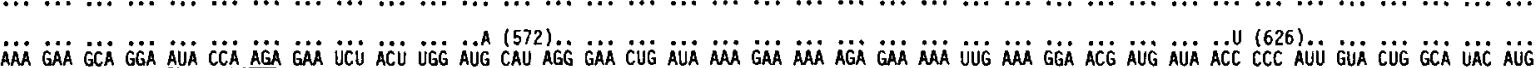
Lys Glu Ala Gly le Pro Arg Glu Ser Thr Trp Met His Arg glu Leu Ile Lys Glu Lys Arg glu Lys Leu Lys Gly Thr Met lle Thr pro Ile Val Leu Ala Tyr Met

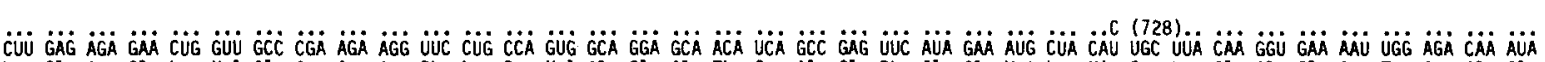
Leu Glu Arg Glu Leu Val Ala Arg Arg Arg phe Leu Pro val Ala Gly Ala Thr Ser Ala Glu phe Ile Glu Met Leu His Cys Leu Gln Gly Glu Asn Trp Arg Gln Ile

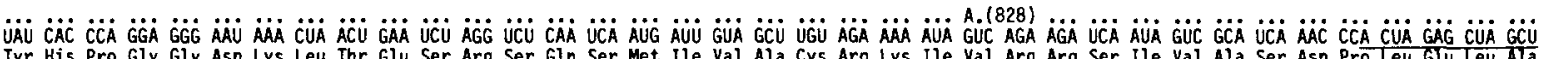
Tyr his Pro Gly Gly Asn Lys Leu Thr Glu Ser Arg Ser Gin Ser Met lle Val Ala Cys Arg Lys nle Val Arg Arg Ser lle val Ala Ser Asn Pro Leu Giu Leu Ala

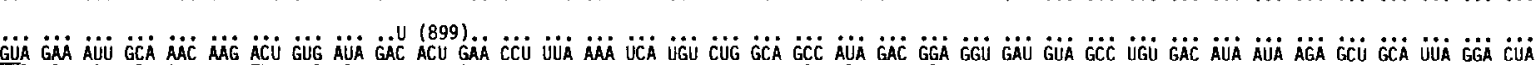
Val Glu Ile Ala Asn Lys thr yal Ile Asp thr Glu Pro Leu Lys Ser cys Leu Ala Ala Ile Asp gly gly Asp Val Ala Cys Asp Jle Ile Arg Ala Ala Leu Gly Leu

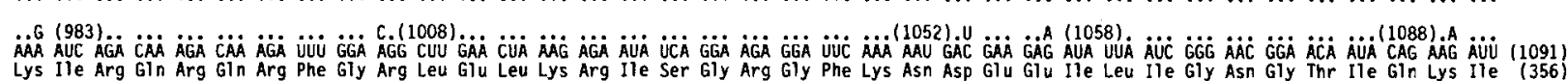
Lys Ile Arg Gln Arg Gin Arg Phe Gly Arg Leu Glu Leu Lys Arg lle Ser Gly Arg Gly Phe Lys Asn Asp Glu Glu lle Leu Ile Gly Asn Gly Thr Ile Gin Lys nle

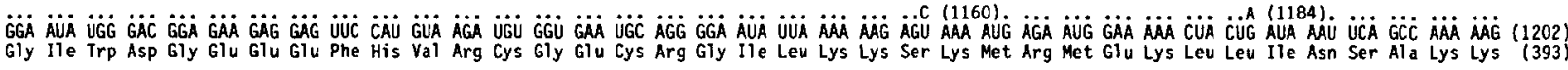

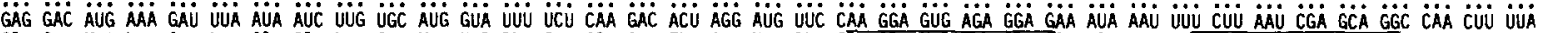
Glu Asp Met Lys Asp Leu Ile Jle Leu Cys Met Val Phe Ser Gin Asp Thr Arg Met Phe Gin Gly Val Arg Gly Glu lle Asn phe Leu Asn Arg Ala Gly Gin Leu Leu (430)

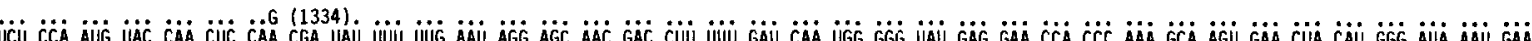
Ser Pro Met Tyr Gln Leu Gin Arg Tyr Phe Leu Asn Arg Ser Asn Asp Leu Phe Asp Gln Trp Gly Tyr Glu Glu Pro Pro Lys Ala Ser Glu Leu His Gly Ile Asn Glu

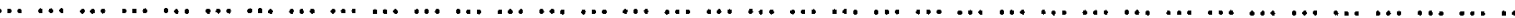

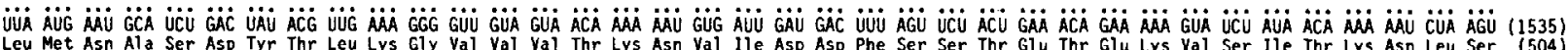
Leu Met Asn Ala Ser Asp Tyr Thr Leu Lys Gly Val Val Wal Thr Lys Asn Val lle Asp Asp Phe Ser Ser Thr Glu Thr Glu Lys val Ser Ile Thr Lys Asn Leu Ser

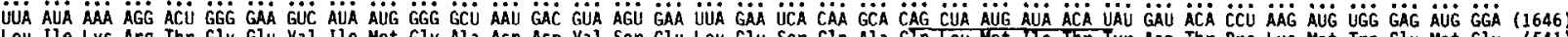
Leu Ile Lys Arg Thr Gly glu Val Ile Met Gly Ala Asn Asp Val Ser glu Leu Glu Ser Gin Ala gin Leu Met Ile Thr Tyr Asp Thr pro Lys Met Trp glu Met Gly (541)

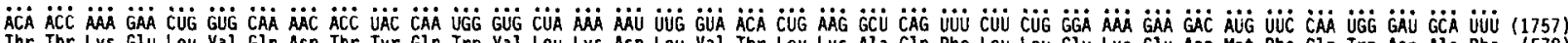
Thr Thr Lys Glu Leu Val Gln Asn Thr Tyr Gin Trp Val Leu Lys Asn Leu Val Thr Leu Lys Ala Gin Phe Leu Leu Gly Lys Glu Asp Met Phe Gln Trp Asp Ala Phe (578)

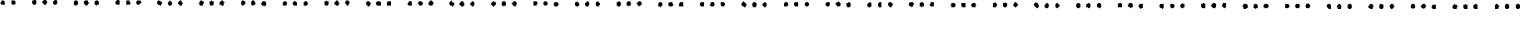

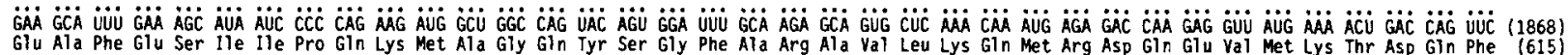
Glu Ala Phe Glu Ser lle fle Pro Gin Lys Met Ala Gly Gin Tyr Ser Gly Phe Ala Arg Ala Val Leu Lys Gin Met Arg Asp Gin Glu val Met Lys thr Asp Gin Phe

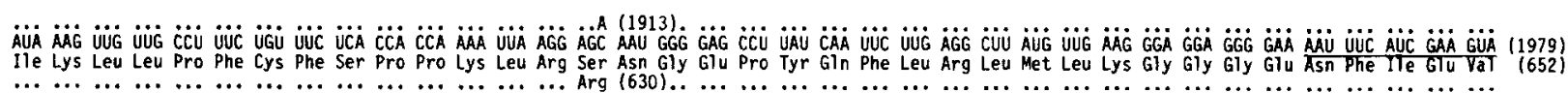

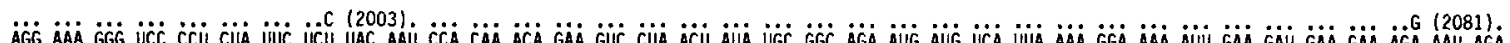

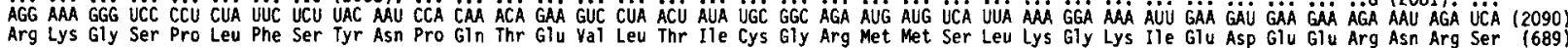

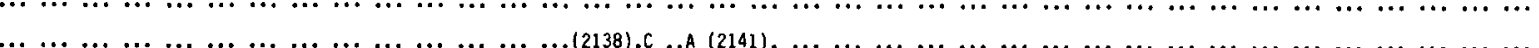

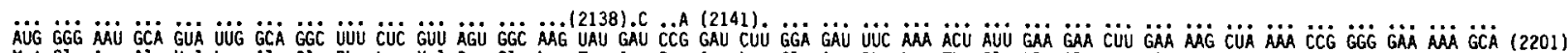
Met Gly Asn Ala Val Leu Ala Gly Phe Leu val Ser Cly Lys Tyr Asp Pro Asp Leu Gly Asp phe Lys Thr Ile Glu Glu Leu Giu Lys Leu Lys Pro Gly Glu Lys Ala (726)

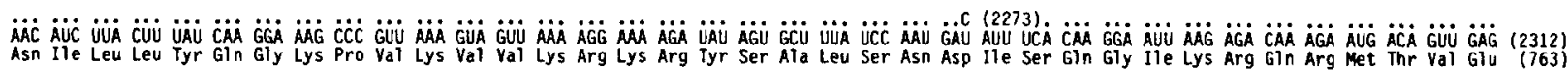

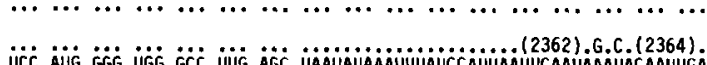

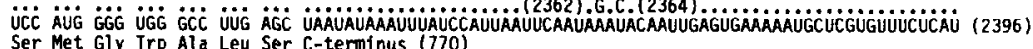
Ser Met Gly Trp Ala Leu Ser C-terminus (770) ...................

FIG. 2. PB2 gene. The ca nucleotide sequence differences are presented above the wt sequence and the amino acid changes are presented below the wt sequence. Each site is followed or preceded by its position number in parentheses and, again, at the end of each line. The position (and unless otherwise noted) the sequence of each oligodeoxynuclcotide primer is indicated by underlining of the wt sequence. A (- - in the sequence represents the deletion of the nucleotide in the corresponding sequence. 


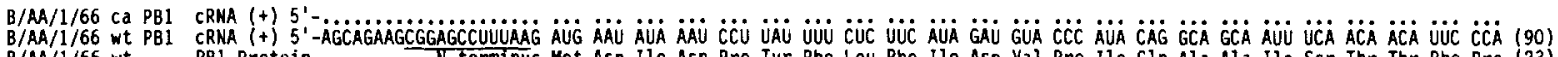
$B / A A / 1 / 66$
$B t$

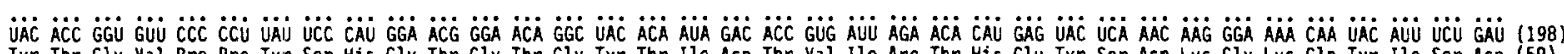
Tyr Thr Giy val Pro Pro Tyr Ser His Gly Thr Gly Thr Gly Tyr Thr Ile Asp Thr val Ile Arg Thr His Gilu Tyr Ser Asn Lys Gly Lys Gin Tyr Ile Ser Asp (59)

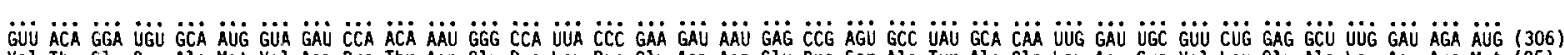
val Thr Gily Cys Ala Met val Asp pro thr Asn gly pro Leu Pro glu Asp Asn glu Pro Ser Ala Tyr Ala gln Leu Asp Cys val Leu glu Ala Leu Asp Arg Met (95)

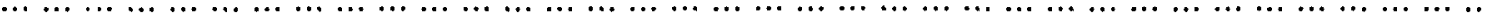

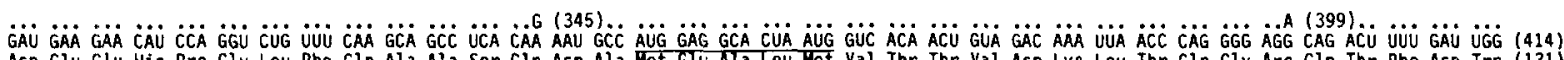
Asp Glu Glu His Pro Gly Leu Phe gln Ala Ala Ser Gin Asn Ala Met Glu Ala Leu het val Thr thr val Asp Lys Leu Thr Gin Gly Arg Gln Thr Phe Asp Irp (131)

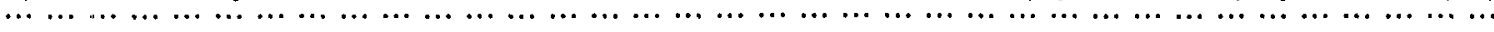

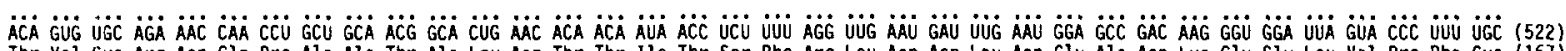
Thr val liys Arg Asn Gln Pro Ala Ala Thr Ala Leu Asn Thr Thr lle Thr Ser Phe Arg Leu Asn Asp Leu Asn Gly Ala Asp Lys gly Giy Leu Val Pro phe Cys (167)

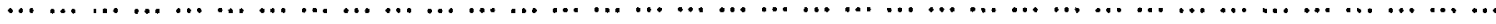

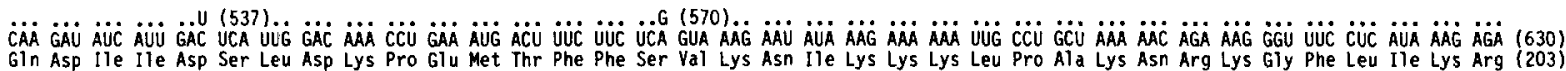

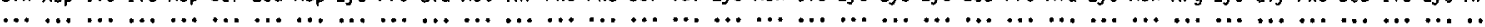

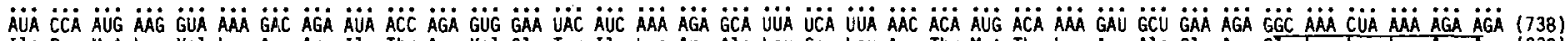
Ile Pro Met Lys val Lys Asp Arg Ile Thr Arg Val Glu Tyr Ile Lys Arg Ala Leu Ser Leu Asn Thr Met Thr Lys Asp Ala Glu Arg GTy Lys Leu Lys Arg Arg $(239)$

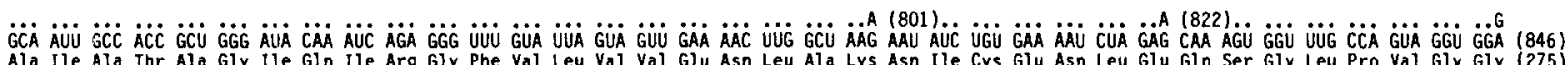
ala He sia thr Alo Gly

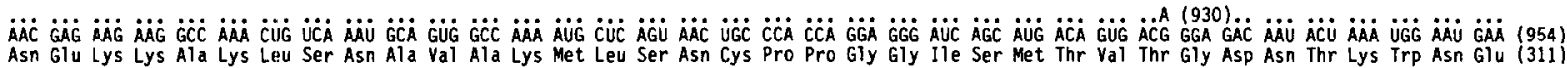
Asn Glu Lys Lys Ala Lys Leu Ser Asn Ala val Ala Lys Met Leu Ser Asn Cys Pro Pro Gly Gly 1e Ser Met $\mathrm{Thr}$ val $\mathrm{Th}$ Gly Asp Asn Thr Lys $\operatorname{trp}$ Asn Glu

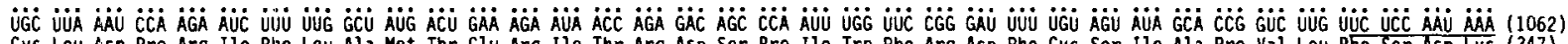
Cys Leu Asn Pro Arg lle Phe Leu Ala Met Thr Glu Arg Ile Thr Arg Asp Ser Pro Ile Trp Phe Arg Asp Phe Cys Ser lle Ala Pro Val Leu Phe Ser Asn Lys (347)

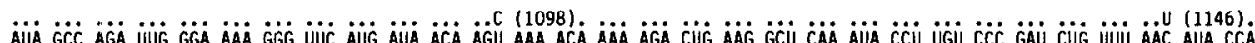

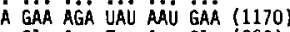

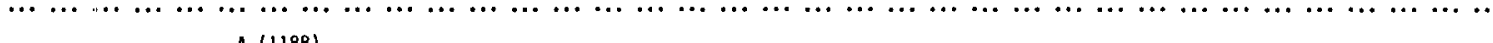

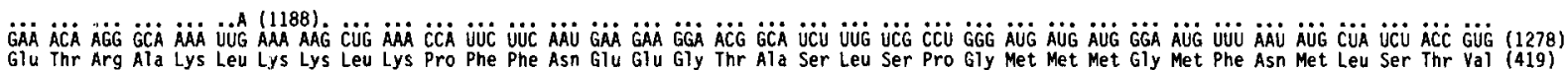
....

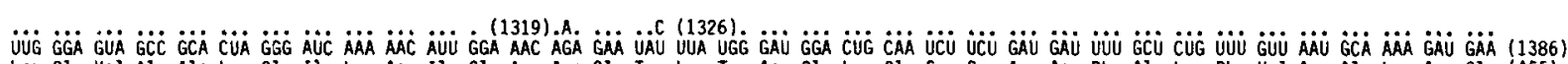
Leu Gly val Ala Ala Leu Giy Ile Lys Asn Ile Gly Asn Arg Giu Jyr Le Trp Asp Gly Leu $6 \ln$ Ser Ser Asp Asp Phe Ala Leu Phe Val Asn Ala Lys Asp Glu (455)

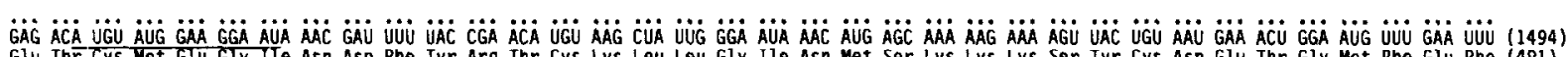

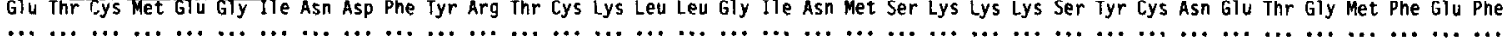

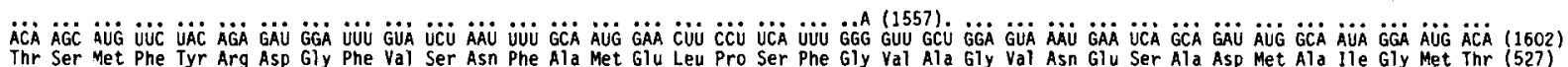

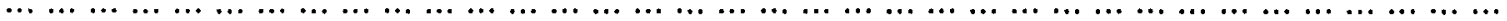

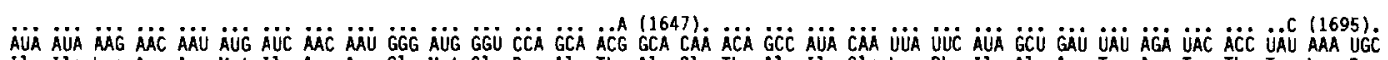

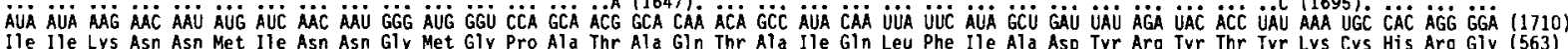
Ile Ile Lys Asn Asn Met Ile Asn Asn Gly Met Gly Pro Ala Thr Ala Gln Thr Ala lle Gin Leu Phe Ile Ala Asp Tyr Arg Tyr Thr $\operatorname{tyr}$ Lys Cys His Arg Gly (563)

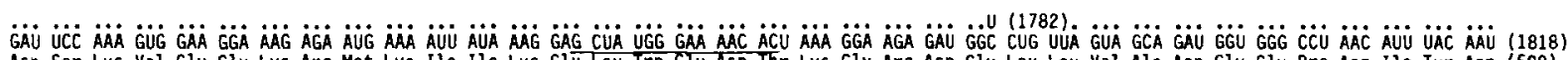
Asp Ser Lys val Glu Gly tys Arg Met Lys Ile Ile Lys Glü Leu Trp Glu Asn Thr Lys Gly Arg Asp gly Leu Leu Val Ala Asp Gly Gly Pro Asn lle Tyr Asn (59g)

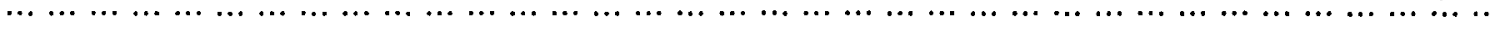

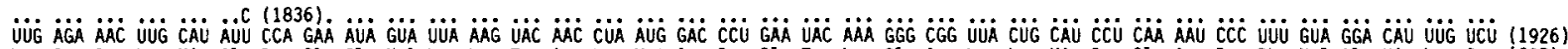
Leu Arg Asn Leu His lle Pro glu Ile Val Leu Lys tyr Asn Leu Met Asp pro Glu Tyr Lys gly Arg leu Leu His Pro gln Asn Pro phe Val Gly His Leu Ser (635)

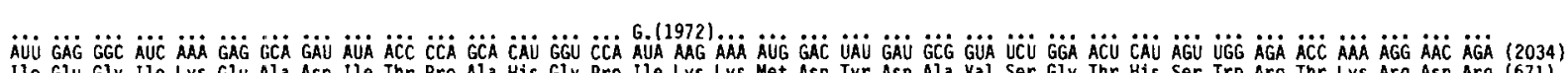

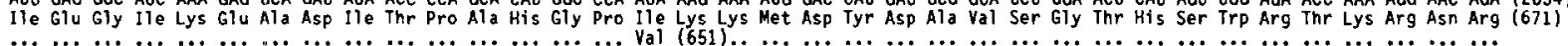

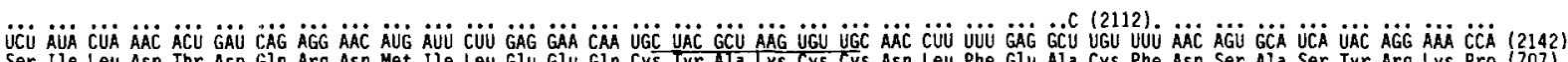
Ser lle Leu Asn Thr Asp Gln Arg Asn Met Ile Leu Glu glu Gin cys Tyr Ala Lys cys cys Asn Leu Phe Glu Ala Cys Phe Asn Ser Ala Ser Tyr Arg Lys Pro (70)

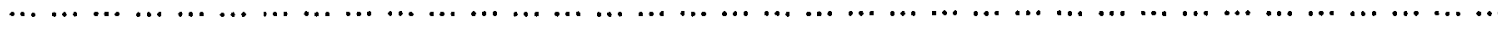

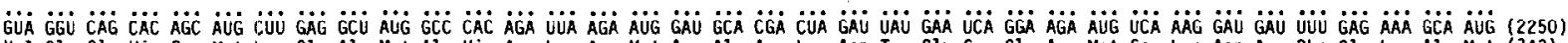
val Gly Gin His Ser Met Leu glu Ala Met Ala His Arg Leu Arg Met Asp Ala Arg Leu Asp Tyr Glu Ser Gly Arg Met Ser Lys Asp Asp Phe glu Lys Ala Met (743)

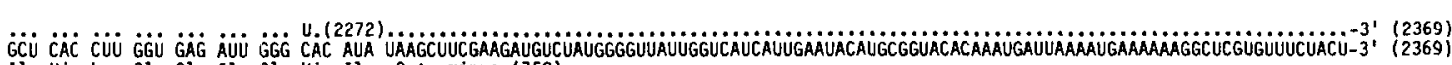
Ala His Leu Gly Glu Ile Gly His Ile C-terminus $(752)$

FIG. 3. PB1 gene. The ca and wt sequences are presented as in Fig. 2, with identical conventions for numbering and primei location and sequences. 


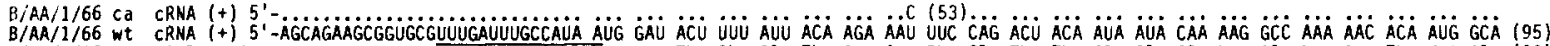

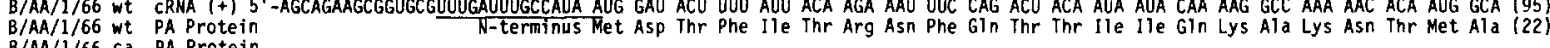

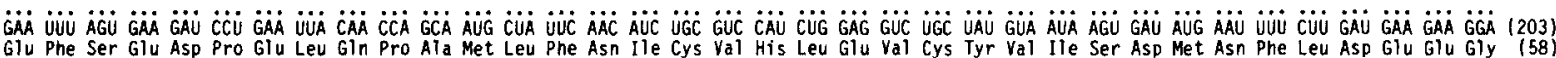

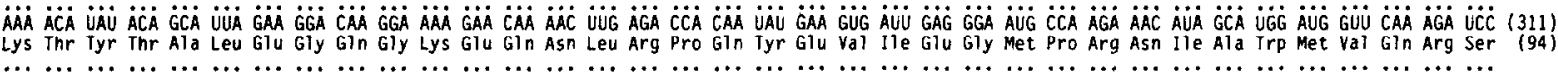

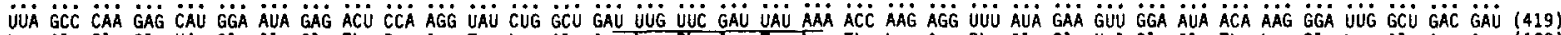
Leu Ala Gin Glu His Gly lle Glu Thr Pro Arg Tyr Leu Ala Asp Leu Phe Asp Tyr Lys Thr Lys Arg Phe lle Glu val Gly Ile Thr Lys Gly Leu Ala Asp Asp (130)

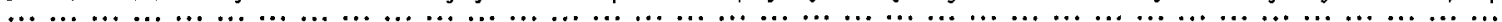

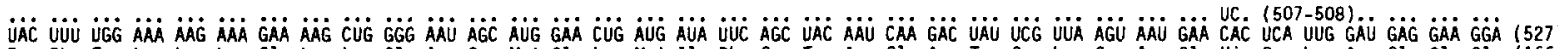
Tyr Phe Trp Lys Lys Lys Glu Lys Leu Gly Asn Ser Met Glu Leu Met lle Phe Ser Tyr Asn Gln Asp Tyr Ser Leu Ser Asn Glu His Ser Leu Asp Glu Glu Gly (166)

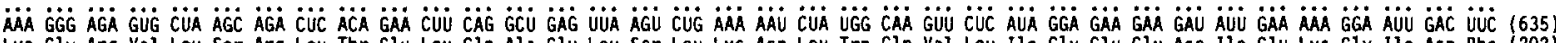
Lys Gly Arg Val Leu Ser Arg Leu Thr glu Leu gin Ala glu Leu Ser Leu Lys Asn Leu Trp gin val Leu Ile Gly Glu Glu Asp Ile glu Lys gly Ile Asp phe (202)

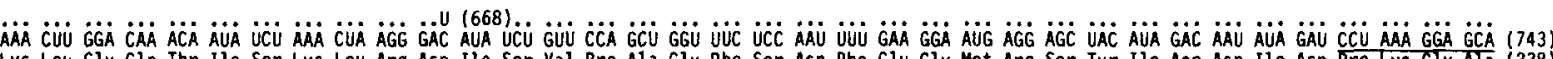
Lys Leu Giy Gln Thr Ile Ser Lys Leu Arg Asp Ile Ser val Pro Ala Gly Phe Ser Asn Phe Glu Gly Met Arg Ser Tyr Ile Asp Asn Ile Asp Pro Lys Gly Ala (238

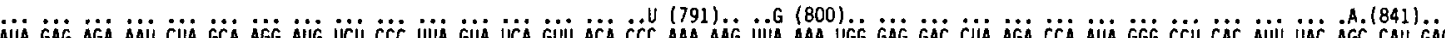
Me Glu Arg Asn Leu Ala Arg Met Ser Pro Leu Val Ser Val Thr Pro Lys Lys Leu Lys Trp Glu Asp Leu Arg Pro lle Gly Pro His Ile Tyr Ser Mis glu Leu (274)

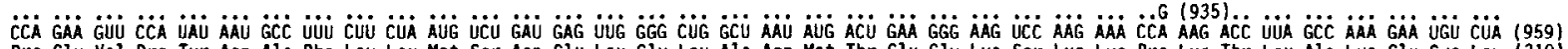
pro Glu Val Pro Tyr Asn Ala Phe Leu Leu Met Ser Asp glu Leu gly Leu Ala Asn Met Thr Glu Gly Lys Ser Lys Lys Pro Lys Thr Leu Ala Lys glu Cys Leu (310)

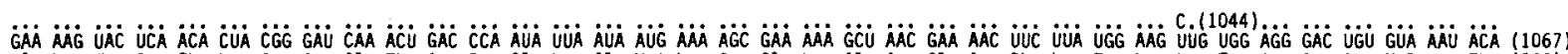
Glu Lys Tyr Ser Thr Leu Arg Asp Gln Thr Asp Pro Ile Leu Ile Met Lys Ser Glu Lys Ala Asn Giu Asn phe Leu Trp Lys Leu Trp Arg Asp Cys Val ASn Thr (346)

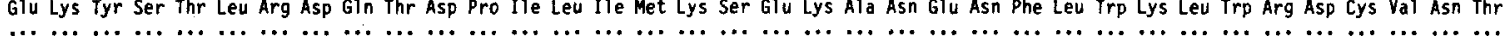

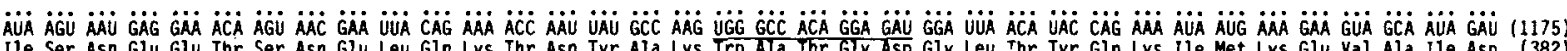
Ile Ser Asn Glu Glu Thr Ser Asn Glu Leu Gln Lys Thr Asn Tyr Ala Lys Trp Ala Thr Gly Asp Gly Leu Thr Tyr Gln Lys Ile Met Lys Glu val Ala Ile Asp (382

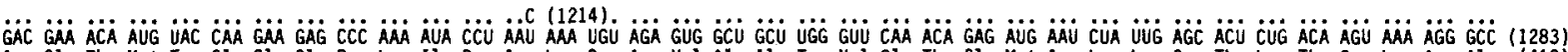
Asp Glu Thr Met Tyr Gin Glu glu Pro Lys Ile pro Asn Lys Cys arg Val Ala Ala Trp val Gin Thr Glu Met Asn Leu Leu Ser Thr Leu Thr Ser Lys Arg Ala (418

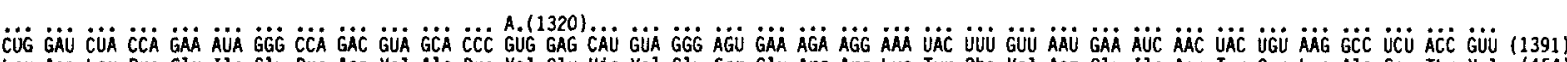

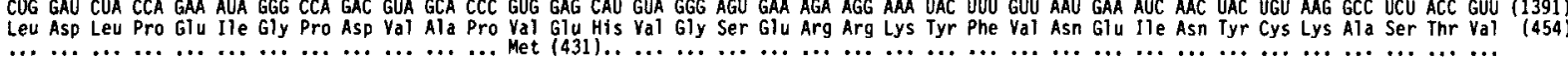

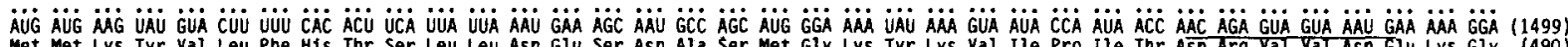
met met Lys Tyr val Leu Phe His Thr Ser Leu Leu Asn Glu Ser Asn Ala Ser Met Giy Lys Tyr Lys val Ile Pro Ile Thr Asn Arg Val VaT Asn Giu Lys Gly (490 gäa äu üui gac aú 6lu Ser Phe Asp lle Leu Tyr Gly Leu Ala Val Lys gly gln Ser His Leu Arg Gly Asp Thr Asp Val Val Thr Val Val Thr phe Glu Phe Ser Ser Thr Asp Pro (526)

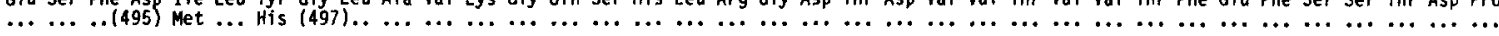

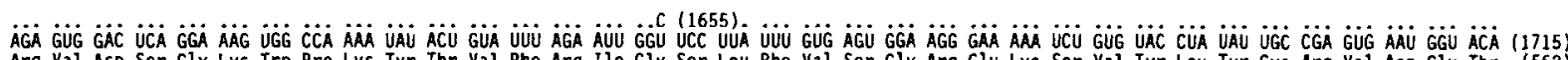
Arg Val Asp Ser Gly Lys Trp Pro Lys Tyr Thr Val Phe Arg lle Gly Ser Leu Phe val Ser Gly Arg Glu Lys Ser Val Tyr Leu Tyr Cys Arg Val Asn Gly Thr (562

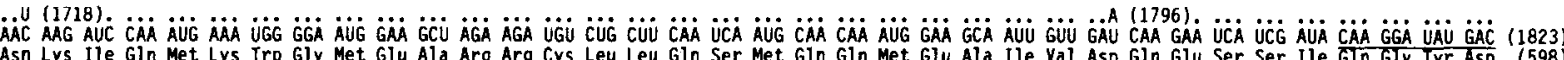

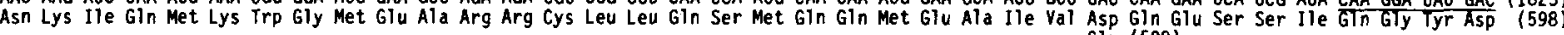

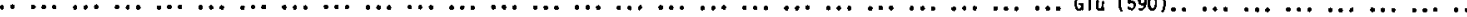

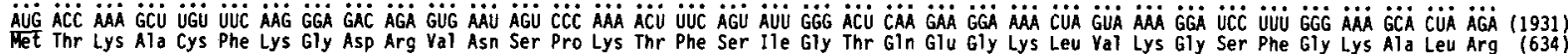

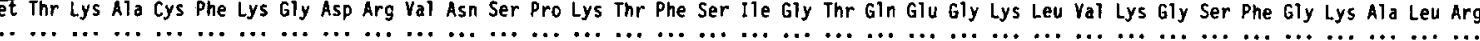

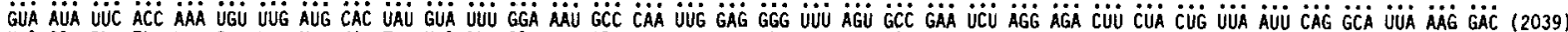
val tle Phe Thr Lys Cys Leu Met His Tyr Val Phe Gly Asn Ala gin Leu Giu Giy Phe Ser Ala Giu Ser Arg Arg Leu Leu Leu Leu Ile Gin Ala Leu Lys Asp (670)

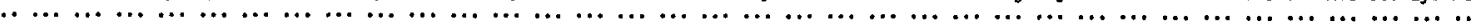

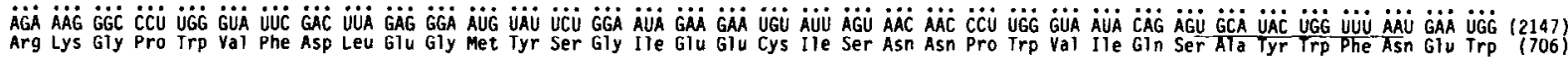

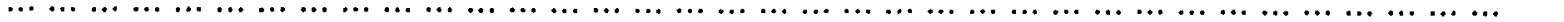

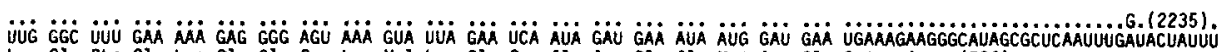
Leu Gly Phe Glu Lys Glu Gly Ser Lys Val Leu Glu Ser Ile Asp Glu tle Met Asp Glu C-terminus (726)

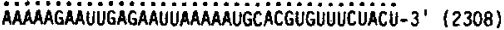

Fig. 4. PA gene. The ca and wt sequences are presented as in Fig. 2, with identical conventions for numbering and primer location and sequences. 


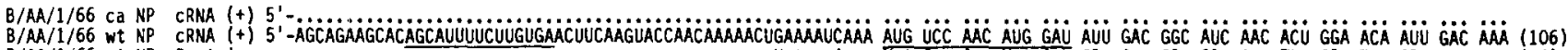
$B / A A / 1 / 66$ wt NP Protein $\mathrm{N}$-terminus het Ser Asn het Asp Ile Asp Gly Ile Asn Thr Gly Thr Ile Asp Lys $(16)$

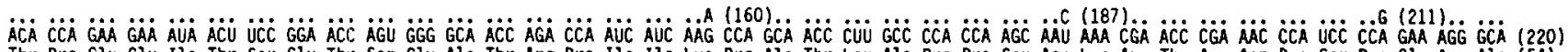
Thr pro Glu Glu Ile Thr Ser Gly Thr Ser Gly Ala Thr Arg Pro lle Ile Lys Pro Ala Thr Leu Ala Pro Pro Ser Asn Lys Arg Thr Arg Asn Pro Ser Pro Glu Arg Ala (54)

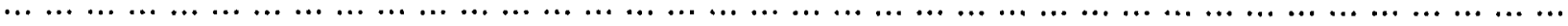

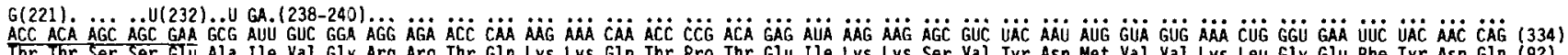
Thr Thr Ser Ser fiu Ala lle Val Gly Arg Arg Thr Gin Lys Lys Gin Thr Pro Thr Glu lle Lys Lys Ser Val Tyr Asn Met Val Vai Lys Leu Gly Glu Phe Tyr Asn Gln (92)

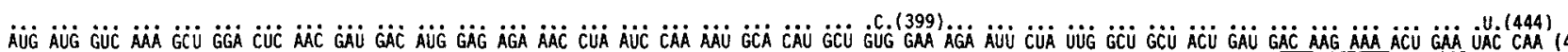
Met Met val Lys Ala Gly Leu Asn Asp Asp Met Glu Arg Asn Leu Ile Gin Asn Ala His Ala Val Giu Arg lle Leu Leu Ala Ala Thr Asp Asp Lys Lys Thr Giu Tyr Gin (130)

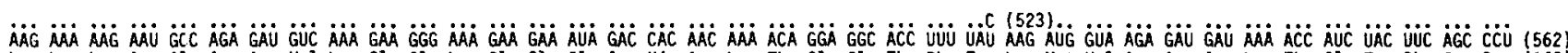
Lys Lys Lys Asn Ala Arg Asp val Lys Glu gly Lys Glu Glu Ile Asp His Asn Lys Thr Gly Gly Thr Phe Tyr Lys Met Val Arg Asp Asp Lys Thr 1le Tyr Phe Ser Pro (168)

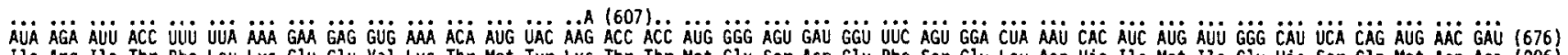
Ile Arg Jle Thr Phe Leu Lys Glu Glu Val Lys Thr Met Tyr Lys Thr Thr Met Gly Ser Asp Gly Phe Ser Gly Leu Asn His Ile Met Ile Gly His Ser Gln Met Asn Asp (206)

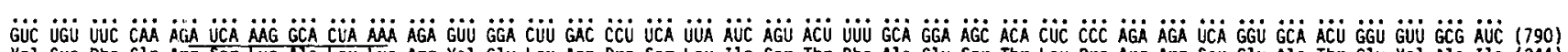
val Cys phe gln Arg Ser Lys Ala Leu Lys Arg Val Gly Leu Asp Pro Ser Leu Jle Ser Thr Phe Ala Gly Ser Thr Leu Pro Arg Arg Ser Gly Ala Thr Gly val Ala Ile (244)

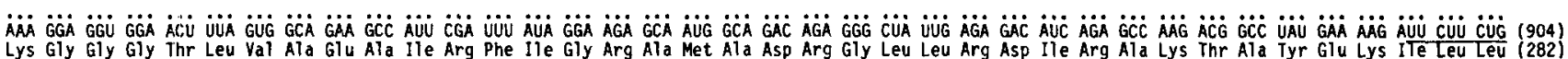

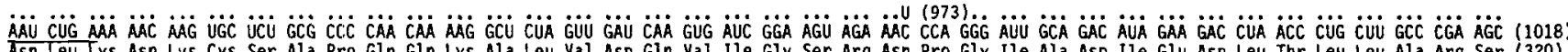
Asn Lys Cys Ser Ala Pro Gln Gin Lys Ala Leu val Asp gin val Jle Gly Ser Arg Asn Pro Gly lle Ala Asp lle glu Asp Leu Thr Leu Leu Ala Arg Ser (320)

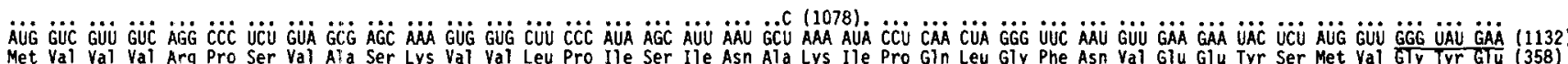

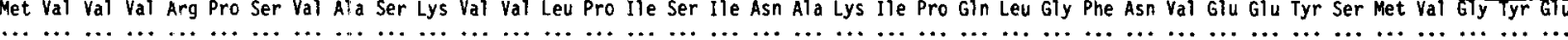

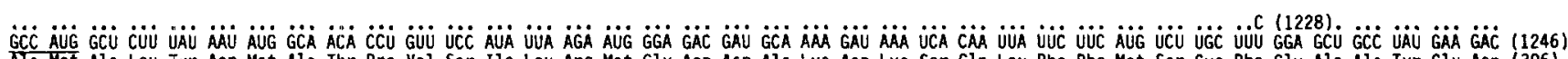
Ala Met Ala Leu Tyr Asn Met Ala Thr Pro val Ser lle leu Arg Met Giy Asp Asp Ala Lys Asp Lys Ser Gln Leu Phe Phe Met Ser Cys Phe Gly Ala Ala Tyr Glu Asp (396)

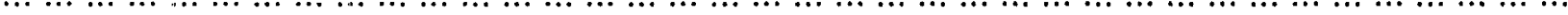

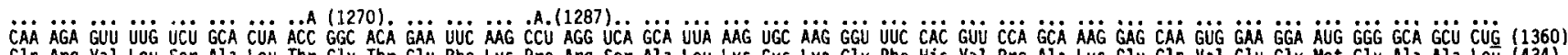
Gin Arg Val Leu Ser Ala Leu Thr Giy Thr Glu Phe Lys Pro Arg Ser Ala Leu Lys Cys Lys Gly phe His Val Pro Ala Lys Glu Gln Val Glu Gly Met Gly Ala Ala Leu (434)

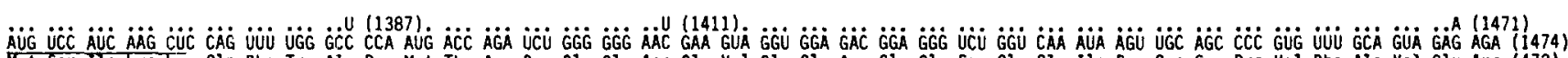
Met Ser lle Lys Leu Gln Phe Trp Ala Pro Met Thr Arg Ser Gly Gly Asn Glu Val Gly Gly Asp Gly Gly Ser Gly Gin Ile Ser Cys Ser Pro Val Phe Rla Yal Glu Arg (472)

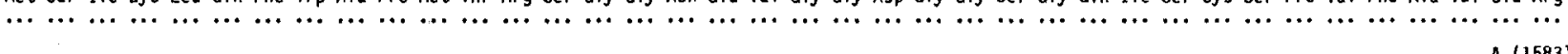

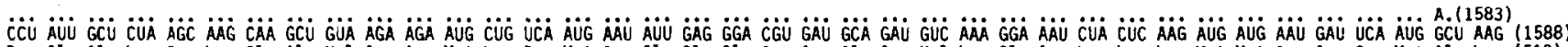
Pro Ile Ala Leu Ser Lys gln Ala Val Arg Arg Met Leu Ser Met Asn Ile Glu Gly Arg Asp Ala Asp Val Lys Gly Asn Leu Leu Lys Met Met Asn Asp Ser Met Ala Lys (510)

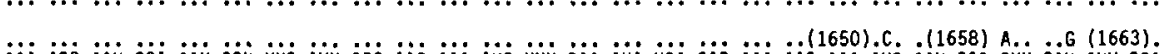

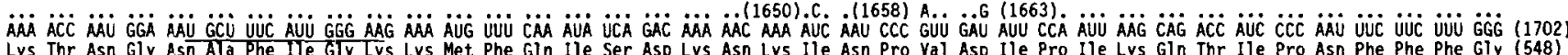
Lys Thr Asn Gly Asn Ala Phe Ile Gly Lys Lys Met Phe Gin 1le Ser Asp Lys Asn Lys Ile Asn Pro val Asp lle Pro 11 Le Lys Gln Thr Ile Pro Asn Phe Phe Phe Gly (548)

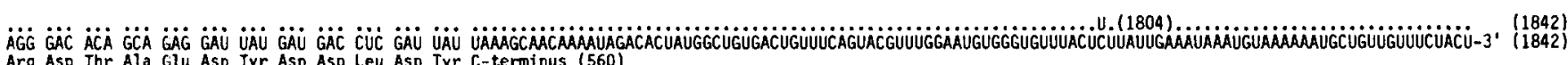
Arg Asp Thr Ala Gilu Asp Tyr Asp Asp Leu Asp Tyr C-terminus $(560)$

Fig. 5. NP gene. The ca and wt sequences are presented as in Fig. 2, with identical conventions for numbering and primer location, with one exception. The primer sequence starting at position 221 is actually ACC ACA AGC AGT GAA (see text).

references;. Both methods were applied directly to VRNA isolated from purified virus. Thus, the determined sequences represent consensus sequences based on the population of vRNA segments. The chemical sequencing method identified the first 50-90 nucleotides of each gene and overlapped the first primer-determined sequence by at least 15 nucleotides. Therefore the sequences on the $5^{\prime}$ side of the first primer positions were determined exclusively by the direct chemical sequencing method. Oligo- deoxynucleotide primers were usually 15 nucleotides in length and the sequence from each preceding primer overlapped the next determined sequence by at least 15-20 nucleotides. Their positions in the sequences are shown by underlining of the wt cRNA sequence of each gene. Only the differences are presented for the ca RNA and predicted ca polypeptide sequences, and these are shown above and below the corresponding wt sequences, respectively. The results for each individual gene are presented below. 


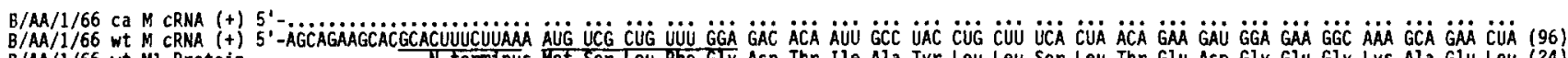

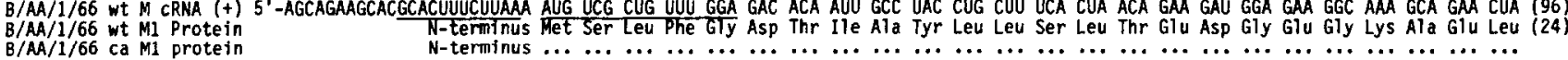

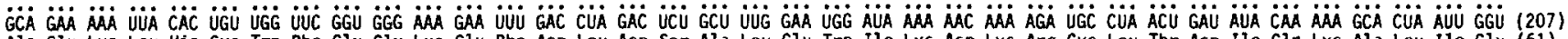
Ala Giu Lys Leu H1s cys Trp phe Gly gly Lys glu Phe Asp Leu Asp Ser Ala Leu Glu Trp Ile Lys Asn Lys Arg Cys Leu Thr Asp Ile Gin Lys Ala Leu 11 e Gly (61)

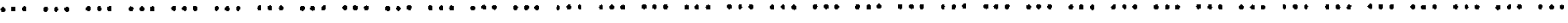

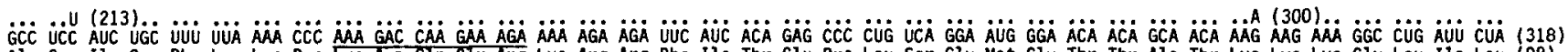
Ala Ser Ile Cys Phe Leu Lys Pro Lys Asp Gin Glu Arg Lys Arg Arg Phe Ile Thr Glu Pro Leu Ser Gly Met Gly Thr Thr Ala Thr Lys Lys Lys Gly Leu Ile Leu (98)

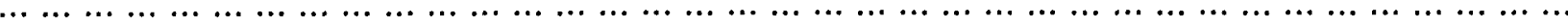

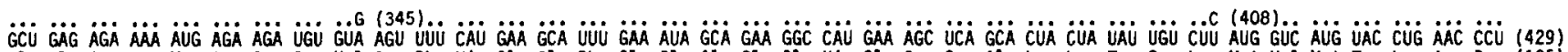

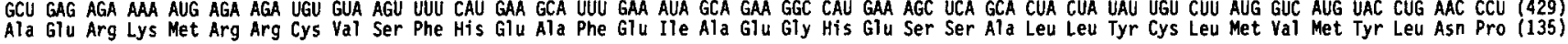

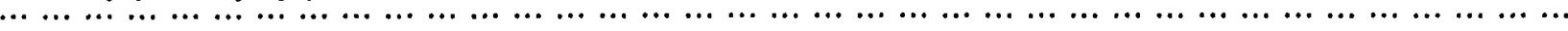

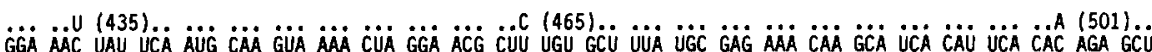

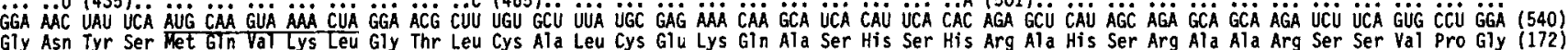
Gly Asn Tyr Ser Met Gin Val Lys Leu Gly Thr Leu Cys Ala Leu Cys Glu Lys Gln Ala Ser His Ser His Arg Ala His Ser Arg Ala Ala Arg Ser Ser val Pro Gly (172)

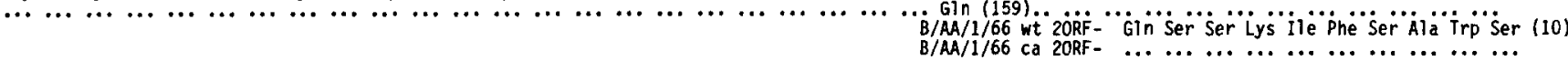

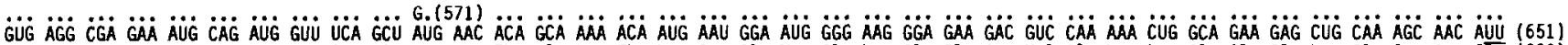
val Arg Arg Glu Met Gin Met Val Ser Ala Met Asn Thr Ala Lys Thr Met Asn Gly Met Gly Lys Gly Glu Asp Val Gln Lys Leu Ala Glu Glu Leu Gin Ser Asn ITe (209)

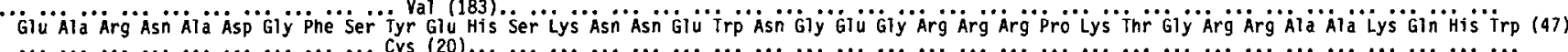

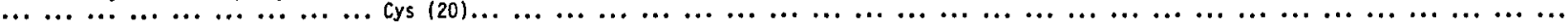

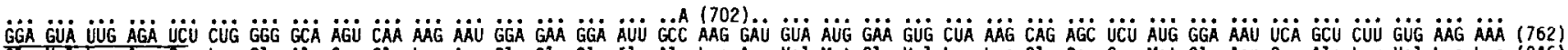
GTy VaT Leu Arg Ser Leu Gly Ala Ser Gin Lys Asn Gly Glu Gly lle Ala Lys Asp val Met Glu Val Leu Lys Gin Ser Ser Met Gly Asn Ser Ala Leu val Lys Lys (246)

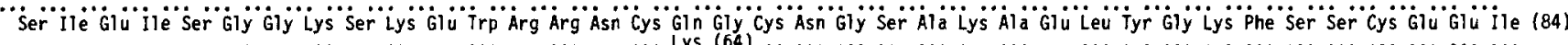

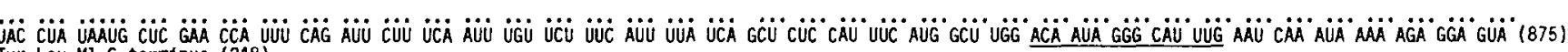
Tyr Leu MI C-terminus $(248)$

Pro lle Met Leu Glu Pro Phe Gin Ile Leu Ser lle Cys Ser Phe Ile Leu Ser Ala Leu His Phe Met Ala Trp Thr Ile Gly His Leu Asn Gin 1le Lys Arg Gly Val (121)

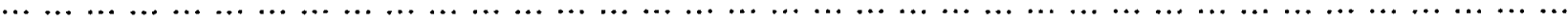

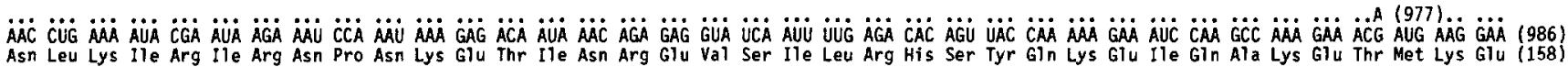

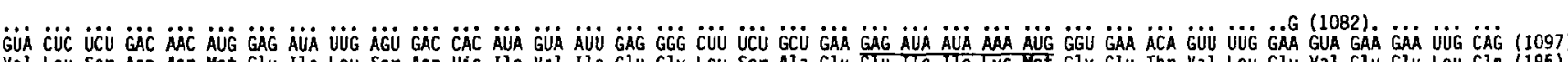
Val Leu Ser Asp Asn Met Glu Ile Leu Ser Asp His Ile Val Ile Glu Gly Leu Ser Ala Glu Glu ITe ITe Lys Met Gly Glu Thr val Leu Glu Val Glu Glu Leu Gin (195)

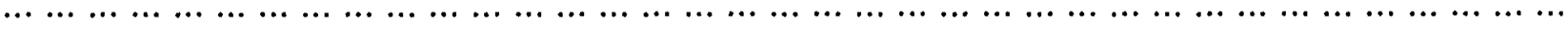

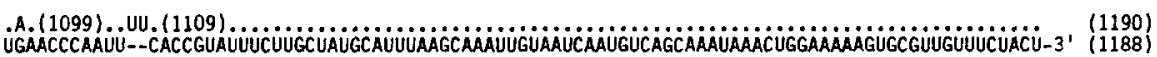
UGAACCCAAUU--CACCG 2ORF C-Terminus
2ORF C-Terminus

FIG. 6. M gene. The ca and wt sequences are presented as in Fig. 2, with the addition the second open reading frame (2 ORF) presented as two additional lines as indicated in the figure.

\section{PB2 gene}

Both wt and ca influen $\angle a$ B/AA1/66 PB2 genes were 2396 nucleotides long. They encoded polypeptides of 770 amino acids. The first methionine codon in the open reading frame began at position 24 (in from the $5^{\prime}$ end of the cRNA) and the first termination codon began at position 2334. A consecutive run of five adenosine residues began at position 2375 and was the probable polyadenylation site. The predicted $w t$ and ca PB2 proteins had molecular weights of 88,035 and $88,072 \mathrm{Da}$, and both had a net charge of +24.5 at $\mathrm{pH} 7.0$, with no large hydrophilic domains. The ca PB2 gene varied from the wt gene by 26 base substitutions. Of these changes, 24 occurred in the coding region. Only 4 of these changes were reflected in amino acid changes. Of these 4 changes, 1 was unique to the ca PB2 gene, and 3 were indentical to sequences noted in other $w t$ influenza $B$ virus $P B 2$ genes (B/Melbourne/43 and $B / A A 1 / 86)$, see Fig. 8. A reassortant containing the $\mathrm{PB} 2$ gene of ca B/AA1/66 virus, and all other genes from a wt virus, $B /$ Houston $/ / 6$, is non-ts, non-ca, and as virulent as the wt virus (Donabedian et al., 1987). Hence, the PB2 gene of the ca donor virus apparently contributed to none of the three phenotypes of interest in an independent manner. It is possible that it may be involved in any of these phenotypes in combination with orle or more other ca B/AA/1/66 genes, especially those involved in the replication complexes.

\section{PB1 gene}

The general characteristics of the influenza B PB1 gene have been previously described by Kemdirim and 
colleagues (1986) for $\mathrm{E} / \mathrm{Lee} / 40$ virus and by DeBorde and colleagues (1987) for B/AA $1 / 66$ wt virus. The ca $B / A A 1 / 66$ PB1 gene varied from the wt PB1 gene by 21 base substitutions all within the coding region of the gene, which resulted in a total of three predicted amino acid changes. By comparison of the ca B/AA/1/ 66 gene sequence to that of B/Lee/40 (Kemdirim et al., 1986) only one of these amino acid changes remained unique to the cold-adapted virus (Fig. 8). From the reassortment analysis of Donabedian and colleagues (1987), PB1, like PB2, apparently played no significant independert role in the establishment of the three phenotypes.

\section{PA gene}

The PA gene sequence has been reported for wt $B / A A / 1 / 66$ virus (DeBorde et al., 1987) and is also known for B/Singapore/79 (Akoto-Amanfu et al., 1987). Our laboratory has also sequenced the PA gene of $B /$ Houston/76 virus. The ca PA gene varied from the wt gene by 17 base substitutions, one of which was in the cRNA $3^{\prime}$-noncoding region. The 16 changes in the coding region resulted in six amino acid changes between the $w t$ and ca PA polypeptides. Comparison with the PA sequences of B/Houston/76 and B/Singapore/ 79 , showed that only two of the six amino acid changes were unique to the ca B/AA $1 / 66$ virus. As shown in the following article (Donabedian et al., 1988 ), the codon of one of these unique positions (position 1320) underwent a change resulting in the reversion of both ts and att phenotypes.

\section{NP gene}

The NP gene has the largest number of base substitutions per nucleotide of any of the six gene pairs examined. Of the 25 changes in the RNA, 24 of them were in the coding region, accounting for nine amino acid changes from wt B/AA/1/66 NP protein to ca B/AA/1/66 NP protein. Comparison of these nine changes with the known NP gene sequences of

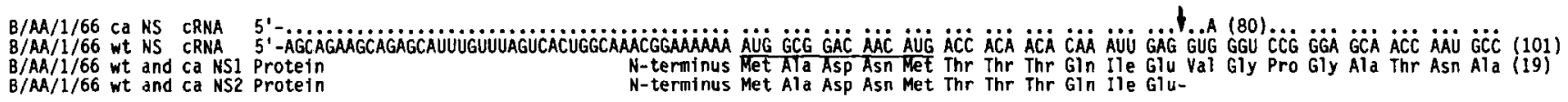

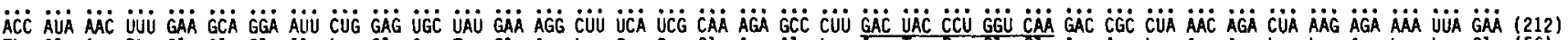
Thr Ile Asn phe Glu Ala Gly Ile Leu Glu Gys Tyr Glu Arg Leu Ser Ser Gin Arg Ala Leu Asp Tyr Pro Gly Gin Asp Arg Leu Asn Arg Leu Lys Arg Lys Leu Glu (56)

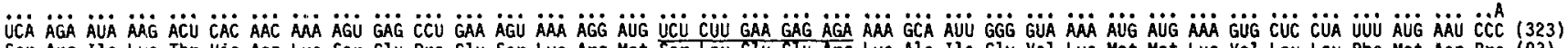
Ser Arg Ile Lys Thr His Asn Lys Ser Glu Pro Glu Ser Lys Arg Met Ser Leu Glu GIU Arg Lys Ala lle Gly Val Lys Met Met Lys Val Leu Leu Phe Met Asn Pro (93)

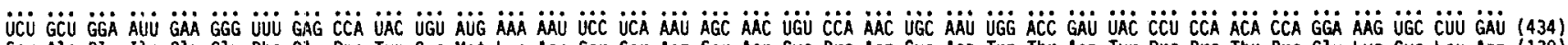
Ser Ala Gly Ile Glu Gly Phe Glu Pro Tyr Cys Met Lys Asn Ser Ser Asn Ser Asn Cys Pro Asn Cys Asn Trp thr Asp Tyr Pro pro Thr Pro Gly Lys Cys Leu Asp (130)

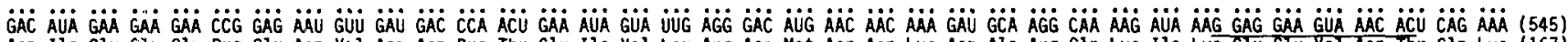
Asp Ile Glu Glu Glu Pro glu Asn Val Asp Asp Pro Thr Glu Ile Val Leu Arg Asp Met Asn Asn Lys Asp Ala Arg Gin Lys Ile Lys Glu Glu VaT Asn Thr Gln Lys (167)

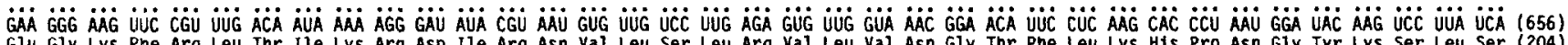

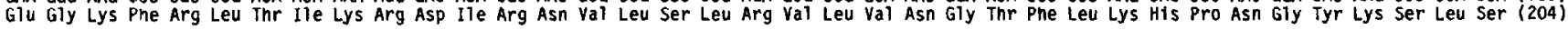

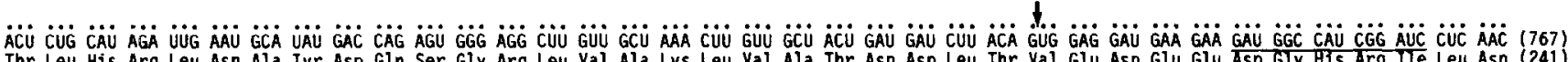
Thr Leu His Arg Leu Asn Ala Tyr Asp Gin Ser Gly Arg Leu Val Ala Lys Leu Val Ala Thr Asp Asp Leu Thr Val Glu Asp Glu Glu Asp Giy His Arg ITe Leu Asn (241)
B/AA/1/66 wt and ca NS2 Protein -Trp Arg Met Lys Lys Met Ala Ile Gly Ser Ser Thr (23)

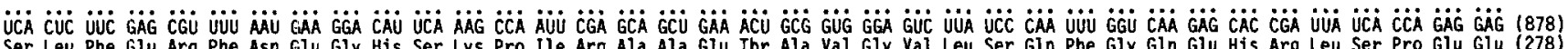
Ser Leu Phe Glu Arg Phe Asn Glu Gly His Ser Lys Pro Ile Arg Ala Ala Glu Thr Ala Val Gly Val Leu Ser Gin Phe Gly Gin Glu His Arg Leu Ser Pro Glu Glu (278)
His Ser Ser Ser Val Leu Met Lys Asp Ile Gin Ser Gin Phe Glu Gin Leu Lys Leu Arg Trp Glu Ser Tyr Pro Asn Leu Val Lys Ser Thr Asp Tyr His Gin Arg Arg (60)

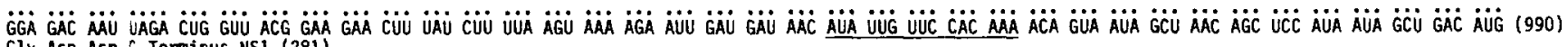

Gly Asp Asn (-Terminus NS1 (281)

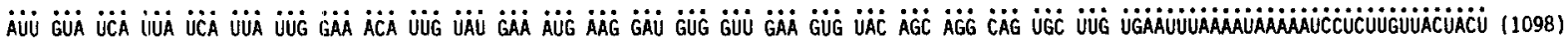

lle val Ser l.eu Ser Leu Leu glu Thr Leu Tyr Glu Met Lys Asp Val Val Glu Val Tyr Ser Arg Gin Cys Leu C-Terminus NS2 (122)

Fig. 7. NS gene. The two nucleotide substitutions in the ca virus NS gene sequence are shown above the wt gene sequence. Because neither base change resulted in an amino acid change, the ca and wt NS1 and NS2 proteins are shown together rather than separately as in the other sequence figures. The arrows represent the presumed splicing sites based on the sequence data given for $B / L e e / 40$ virus (Briedis and Lamb, 1982). 


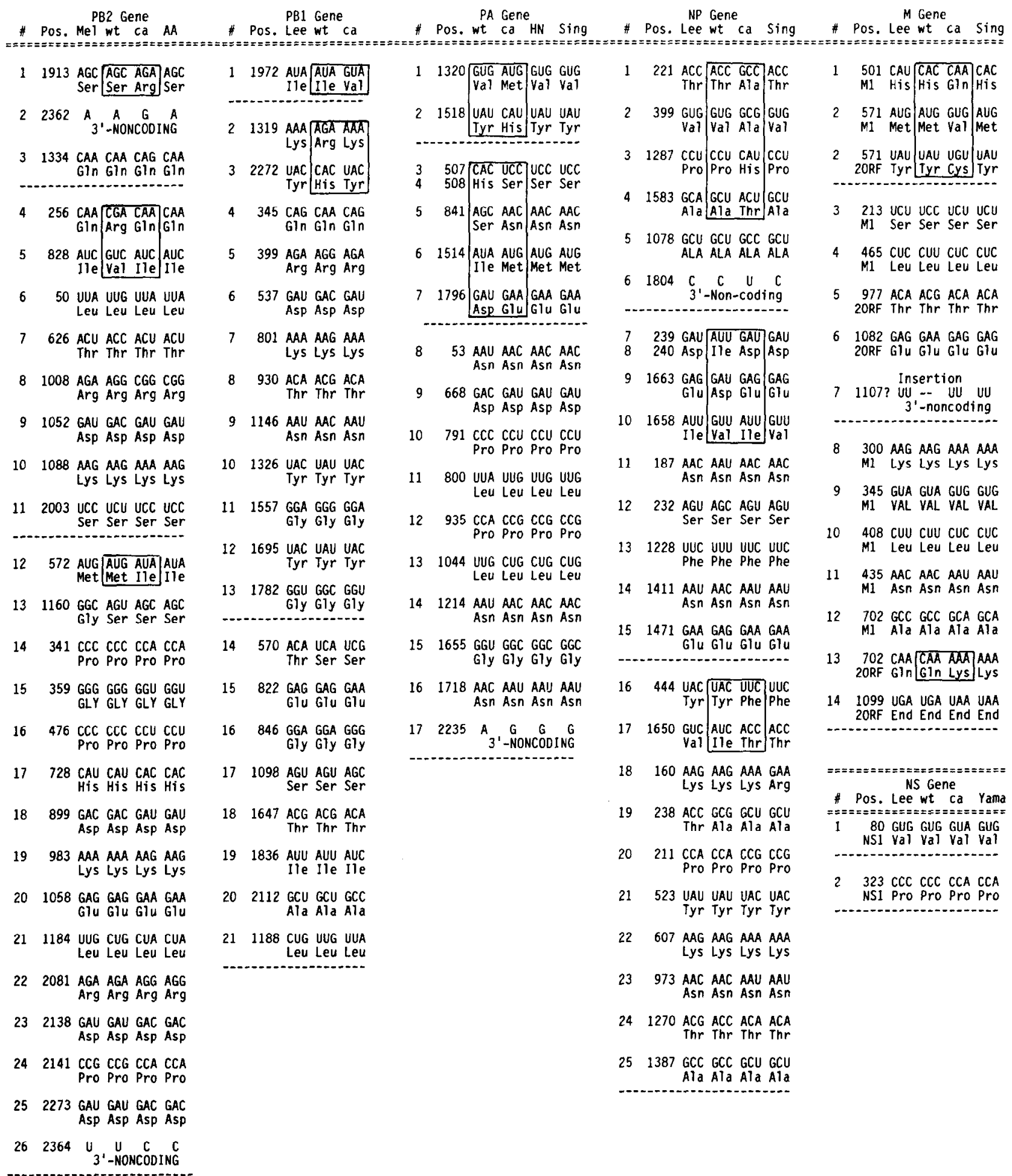

Fig. 8. Unique and nonunique wt to ca changes. The sequence differences between the wt and ca B/AA/1/66 nonsurface genes are compared to sequences of other known influenza type B viruses. They are presented as part of the codon of which they are a member, rather than just a single nucleotide difference. The encoded amino acid is listed below the codon. Below each gene the sites are arranged as follows: changes unique to $\mathrm{ca} B / \mathrm{AN} 1 / 66$ followed by changes that match the sequence in one or more other influenza type $B$ virus. These nonunique changes have been further grouped by matching to one or another known viral sequence. The columns labeled Pos. refer to the nucleotide position of the change as found in Figs. 2-7. I hose nucleotide difterences that result in amino acid differences have been enclosed in boxes. The viruses listed are as follows: Mel, B/Meibourne/43; Lee, B/Lee/40; HN, B/Houston/76; Sing, B/Singapore/222/79; AA, B/Ann Arbor/1/86; wt, wt B/AA $1 / 66$; and $\mathrm{ca}$, ca B/AA $1 / 66$. 
B/Lee/40 (Briedis and Tobin, 1984) and B/Singapore/ 72 (Londo et al., 1983) left only four of these changes as unique to the ca NP gene (Fig. 8).

The primer sequence starting at position 221 is actually ACC ACA AGC AGT GAA. Thus its sequence varies from that of the wt sequence at position 232 , and from that of the ca sequence at position 221 . This primer was made based on $\mathrm{B} /$ Lee $/ 40$ sequences, but despite the one nucleotide difference for each $\mathrm{B} / \mathrm{AA} 1 / 66$ virus, it worked well. (All other primers which were made prior to actual B/AA/1/66 viral sequence knowledge and which were later shown to vary by even one nucleotide, would not function.)

\section{M gene}

7 here was a total of 12 nucleotide substitutions between wt and ca B/AA/1/66 M RNA. In addition, the ca $B / A A / 1 / 66 \mathrm{M}$ gene had an insertion of two uridine nucleotides within positions 1107-1110. The insert position was ambiguous due to the presence of two uridine nucleotides at positions 1107-1108 in the wt $B / A A / 1 / 66 \mathrm{M}$ gene. This insertion was in the 3 'noncoding region for $M_{1}$ protein and was also beyond the conserved second open reading frame (2 ORF) previously described for both $\mathrm{B} / \mathrm{Lee} / 40$ (Briedis et al., 1982) and B/Singapore/222/79 (Hiebert et al., 1986) M genes. In fact, both of these viruses have a run of four uridine nucleotides at this position like the ca $\mathrm{M}$ gene. There were two amino acid changes in the $M_{1}$ protein region, and two predicted amino acid changes in the possible product of the 2 ORF. None of the $M_{1}$ changes are found in the other known influenza $B M$ gene sequences, while one of the changes in the 2 ORF is also seen in B/Singapore/222/79 M gene (Hiebert et al., 1986). Thus, at this time only the two changes in the $M_{1}$ protein and possibly one in the 2 ORF appeared to be unique to the cold-adapted virus (Fig. 8).

\section{NS gene}

The NS gene had the lowest number of changes between wt and ca B/AA/1/66 viruses. Only two base substitutions were present, both exclusively in the $\mathrm{NS}_{1}$ coding region, and neither of these resulted in amino acid changes in the $\mathrm{NS}_{1}$ protein. One change, at position 80, occurred in the $5^{\prime}$-donor-splice region, but it was an acceptable change based on the consensus signal from Mount (1982). Unless the wt NS gene itself was inherently attenuating, cold-adapted, or temperature-sensitive, we would predict that this gene contributes little to the overall phenotypes of the ca B/AA1/ 66 virus.

\section{Unique changes of $\mathrm{ca} B / A A / 1 / 66$ virus}

The total nucleotide and amino acid changes between the six nonsurface genes of wt and $\mathrm{ca} B / A \mathrm{AA} / 1$ 66 viruses are given in Fig. 8 showing a comparison to the corresponding site in other known influenza $B$ sequences. Except for those changes which occur in the noncoding regions of the genes, the sites are presented in their codon, with their encoded amino acid. Where possible the changes have been divided into groups: (1) those nucleotide changes that were unique to ca B/AA $1 / 66$ virus (the top group for each gene), and (2) those changes that were also seen in one or more other wt viruses (the bottom two groups for each gene). Sequences for PB2, NP, M, and NS genes have been determined from viral isolates that occurred both earlier and later in time than the B/AA $1 / 66$ viruses. Only one other sequence for the PB1 gene has been reported to which $B / A A 1 / 66$ viral $P B 1$ sequences can be compared, and it was determined from an earlier virus isolate $(B / L e e / 40)$. Therefore, the group of changes, PB1 positions 14-21, may contain some unique ca nucleotide changes even though no amino acid change results. The PA gene has two other PA sequences to which it can be compared, but both genes are from later viral isolates. The nucleotide changes that result in amino acid changes are enclosed in boxes. Out of 15 unique nucleotide changes, 10 of these resulted in 11 unique amino acid changes. (One change in the $\mathrm{M}$ gene caused a unique amino acid change both in $M_{1}$ and in the 2 ORF.) The 89 remaining nonunique nucleotide changes resulted in only 15 additional amino acid changes.

\section{Total changes}

Table 1 presents a summary of the total numbers of nucleotide and amino acid changes that were selected in the cold-adaptation of wt B/AA $1 / 66$ virus. The lack of changes occurring in the $5^{\prime}$-noncoding regions was probably not indicative of an active conservation of sequence, since overall, only one or two changes would have been predicted by the observed, average rate of change: i.e., 199 nucleotides/5'-noncoding region $\times 0.0094$ changes $/$ nucleotides $=1.88$ changes $/$ 5 -noncoding region. More disturbing is the lack of change in the NS gene. While all other genes range around 0.1 change/nucleotide, that of the NS gene is fivefold less. The reasons for this difference are not known. Only 26 amino acid changes are predicted to occur from a total of 105 nucleotide changes. This indicates a high degree of functional constraint, since on a random basis, 105 changes should result in approximately two-thirds as many amino acid changes, 
TABLE 1

Summary of B/AA1/66 wt to ca Sequence Changes

\begin{tabular}{lccccc}
\hline & \multicolumn{5}{c}{ CRNA } \\
\cline { 2 - 6 } & $5^{\prime}-$ & Coding region & $-3^{\prime}$ & Total & $\%$ \\
\hline PB2 & $0 / 23$ & $24 / 2310$ & $2 / 63$ & $26 / 2396$ & $(1.11)$ \\
PB1 & $0 / 21$ & $21 / 2256$ & $0 / 92$ & $21 / 2369$ & $(0.89)$ \\
PA & $0 / 29$ & $16 / 2178$ & $1 / 101$ & $17 / 2308$ & $(0.74)$ \\
NP & $0 / 58$ & $24 / 1680$ & $1 / 104$ & $25 / 1842$ & $(1.36)$ \\
M & $0 / 24$ & $12 / 1073^{3}$ & $2 / 93$ & $14 / 1190$ & $(1.18)$ \\
NS & $0 / 44$ & $2 / 1021^{\circ}$ & $0 / 33$ & $2 / 1098$ & $(0.18)$ \\
Total & $0 / 199$ & $99 / 10,518$ & $6 / 486$ & $105 / 11,203$ & $(0.94)$ \\
& \multicolumn{5}{c}{ Protein } \\
\end{tabular}

\begin{tabular}{|c|c|c|}
\hline & Total changes/length & Unique changes/length \\
\hline PB2 & $4 / 770 \quad(0.52 \%)$ & $1 / 770 \quad(0.13 \%)$ \\
\hline PB1 & $3 / 752 \quad(0.40 \%)$ & $1 / 752 \quad(0.13 \%)$ \\
\hline PA & $6 / 726 \quad(0.83 \%)$ & $2 / 726 \quad(0.28 \%)$ \\
\hline NP & $9 / 560 \quad(1.61 \%)$ & $4 / 560 \quad(0.71 \%)$ \\
\hline M1 & $2 / 248 \quad(0.81 \%)$ & $2 / 248 \quad(0.81 \%)$ \\
\hline $2 \mathrm{ORF}^{\mathrm{C}}$ & $2 / 195 \quad(1.03 \%)$ & $1 / 195 \quad(0.51 \%)$ \\
\hline NS1 & $0 / 281 \quad(0 \%)$ & $0 / 281 \quad(0 \%)$ \\
\hline NS2 & $0 / 122 \quad(0 \%)$ & $0 / 122 \quad(0 \%)$ \\
\hline Total & $26 / 3654(0.71 \%)$ & $11 / 3654(0.30 \%)$ \\
\hline
\end{tabular}

$a, b$ Coding region length includes both open reading frames.

${ }^{\circ} 2$ ORF, Second open reading frame, and length is given for second open reading frame.

i.e., 71. Of the 26 total amino acid differences that exist between the six pairs of wt and ca genes, only 11 are unique to ca $\mathrm{B} / \mathrm{AA} / 1 / 66$ virus.

\section{DISCUSSION}

As seen in Fig. 1, the initial $\mathrm{CK}_{1}$ isolate of wt $B / A A / 1 / 66$ virus had not been cloned by plaquing prior to the cold-adaptation process. Therefore, it was most likely a pool of viruses whose individual genomic sequences were highly related, but to some extent different for each virus. This assumption is based on the data of Parvin and colleagues (1986) which give a mutation rate of $1.5 \times 10^{-5}$ mutations/nucleotide/generation for influenza A virus. At this rate of mutation, they estimate that every virion in a virus pool will vary by an average of one mutation per genome after five generations. The further removed in time a virus line is from its last plaquing step, the more diverse its individual viruses. Thus, when a virus pool is sequenced directly using either dideoxynucleotide chain-termination or chemical methods and purified VRNA, it is a consensus sequence that is determined and not the absolute sequence of a particular virus.
It should be noted that mutations which occur during every generation of the virus become fixed in the dominant line (and hence appear in the consensus sequence) only if they are in the infecting virus selected by a plaquing step, or if they confer a very great growth advantage compared to the standard population. Mutations which confer growth advantages at suboptimal temperatures have a better chance of predominating in the population in the cold adaptation process, and hence a better chance of becoming selected during plaquing. In addition to these selected mutations, nonlethal mutations may also be selected at random by each plaquing step. Thus, in comparing the final two populations of $\mathrm{ca}$ and wt $\mathrm{B} / \mathrm{AA} 1 / 66$ vi ruses, the sequence changes that exist will primarily represent (1) sequence differences existing between the particular wt virus, that was the progenitor of the ca virus, and the wt virus consensus sequence, (2) cold-selected mutations (these differences may have already existed in the wt pool or have occurred spontaneously during the cold-adaptation process and then been selected, and (3) other spontaneous nonlethal mutations that occur randomly with each generation, and become fixed by the mechanism of plaquing as part of the final ca virus consensus sequence.

Thus the cold-adaptation procedure and the plaquing procedures not only selected changes that resulted in the ca, ts, and att phenotypes, but also at the time of the first plaquing, a particular individual virus was selected from the mixed wt pool that would inherently contain a number of sequence differences from the consensus sequence of the wt virus pool, which probably have no bearing on these three phenotypes. It is conceivable that a change in the nucleic acid sequence might enable the expression of the ca phenotype without a corresponding amino acid change, but it is more likely that the cold-adaptation procedure operates by selecting viruses whose proteins function better at the low temperature. Thus, those nucleotide changes that were cold-selected should most likely be reflected in an amino acid change. This condition certainly fits the set of 15 unique nucleotide changes, since they were responsible for 11 of the 26 total amino acid changes. The other 15 amino acid changes were encoded by a subset of the other 90 nonurique nucleolide changes. While it is impossible to go back now and determine which changes occurred at which stage in the cold adaptation process, it is very likely that a large part of the unique changes represented cold-selected changes, while most nonunique changes represented nonlethal random site differences that existed between the wt consensus 
sequence and an individual wt virus that was the progenitor of the ca virus.

The nonunique changes can be ordered into groups as mentioned earlier, in which the ca sequence remains the same as that of another wt virus. Sequences for the PB2, NP, M, and NS genes have been determined from isolates which bracket the B/AA $1 / 66$ sequences in time. Thus in these genes it can be determined if the ca virus sequence change resembles that of an earlier or later virus. It should be mentioned that in those sites where the ca sequence is identical to an earlier virus, except in one instance (NP gene, position 1658 ) it is identical to a later virus as well, with the wt $\mathrm{B} / \mathrm{AA} 1 / 66$ sequence being unique. There are 22 sites in which the ca sequence is identical to an earlier virus, and 33 sites in which the ca sequence resembles that of a later virus for these four genes. Because $B / A A 1 / 66$ ca virus is not an evolutionary progenitor to the later viruses, these changes either existed between the sequence of the individual wt virus that was to be the progenitor of the ca virus and the wt consensus sequence prior to the cold-adaptation process, or there is some unknown mechanism which causes convergent changes at specific sites. This latter possibility is unlikely for two reasons: (1) Convergent changes might appear to happen if some sites have a higher than normal rate of mutation and simply switch back and forth between purine to purine, or pyrimidine to pyrimidine, manifesting either as one or the other depending upon the isolate sequenced. However, if this were the case, then out of the 22 sites in which the ca virus sequence is identical to the earlier virus sequence, a good number should show the wt B/AA $1 / 66$ site identical to the later virus isolate's sequence. As mentioned above, this sequence configuration actually occurred only once in 22 times. (2) True convergent change implies that all the changes that appeared in the ca virus sequence occurred as mutations to the wt sequence. Based on the mutation rate determined previously for influenza $A$ virus (Parvin et al., 1986), the 55 differences for these four internal protein genes could not have arisen during the limited number of passages that occurred during the cold-adaptation and plaquing process. From the original wt isolate there were seven chick kidney passages, nine egg passages, and seven plaquing steps to arrive at the virus pool responsible for the ca consensus sequence. Estimating five generations for each of these steps as in Parvin and colleagues (1986), we would calculate 23 steps $\times 5$ generations $/$ steps $=115$ generations. Using the mutation rate of $1.5 \times 10^{-5}$ mutations/nucleotide/generation (Parvin et al., 1986), and a total of 6526 nucleoticles for the PB2, NP, M, and NS genes, we can determine the total number of mutations expected to have arisen during the cold-adaptation procedure: $1.5 \times 10^{-5}$ mutations/nucleotide/generation $\times 6526$ nucleotides $\times 115$ generations $=11.26$ mutations expected to have occurred during the ca selection and plaquing procedure, not 55 changes. This mutation rate was determined for influenza $A$ virus. Comparison of the rate of change for influenza $A$ and $B$ viruses had previously indicated that influenza $A$ has a higher rate of change than influenza $B$ virus, by as much as fivefold for known HA gene sequences (Palese and Young, 1983). Thus, it is even more unlikely that all 55 mutations occurred during the cold-adaptation process. Thus, while some of the nonunique nucleotide changes that exist between the wt and ca B/AA/ $1 / 66$ viruses may be accounted for by mutations that occurred during the ca process, they would be predicted to account for only a small percentage of the number actually observed. The mechanism of convergent mutation cannot be entirely ruled out, however, since the number of viral generations in man prior to isolation are not known, and the convergent changes could have occurred then.

Of the six genes examined, the NS gene was quite restricted in the rate of change/nucleotide from the other five genes. If this restriction were due to a functional constraint inherent to the NS gene, then a similar restriction might be observed between wt NS genes as well. The NS gene of $B /$ Lee/40 is 1096 nucleotides long. Both $\mathrm{ca}$ and wt B/AN1/66 NS genes had an additional two nucleotides due to the insertion of two additional adenosine residues within a run of three existing adenosine residues at positions 39 to 41 of the $\mathrm{B} / \mathrm{Lee} / 40$ sequence. This two-nucleotide insertion occurred in the $5^{\prime}$-noncoding region of MRNA and does not affect the NS1 and NS2 protein sequences. In addition to the two unmatched adenosine residues, there are 61 base mismatches between the $B / A A / 1 / 66$ $w t$ virus and $B / L e e / 40$ virus. This amount of change is roughly the same as that found between the other known genes of $\mathrm{B} / \mathrm{Lee} / 40$ and $\mathrm{B} / \mathrm{AA} 1 / 66$ viruses (DeBorde et al., 1987), indicating that the minimal nucleotide change occurring between wt and ca B/AA/1/66 NS gene pair is probably not due to an inherent functional restriction in the NS gene, but to some other reason. Whether it is specific to cold-adaptation or random chance is not known.

Genetic studies utilizing reassortants that were made between ca $B / A A / 1 / 66$ and wt $B / H N / 76$ have indicated that the PA gene is the primary determinant of the ts and att phenotypes (Donabedian et al., 1987). A combination of other ca B/AA/1/66 genes (without the PA gene) could also contribute to attenuation. $A$ 
reassortant that contained the NP gene from ca $\mathrm{B} / \mathrm{AA} / 1 / 66$ virus and all other genes from $\mathrm{B} / \mathrm{Texas} / 1 / 84$ was shown to be ca and ts in PCK cells, and partially attenuated in ferrets (unpublished data, this laboratory). In the study of Donabedian and colleagues (1987) no single-gene NP reassortant was isolated, but reassortants containing the NP and other genes from $B / A A 1 / 66$ ca virus, without the PA gene, were shown to have a $2-\log _{10}$ reduction in growth at $39^{\circ}$ compared to growth at $33^{\circ}$. One of these reassortants is also restricted for viral growth in ferret lungs, a probable marker for attenuation (Donabedian et al., 1987). Thus the NP gene may also carry mutations involved in the final level of ts and att phenotypes of $\mathrm{ca} B / A \mathrm{~A} / 1 / 66$ virus and its ca vaccine reassortants. From the studies of the reassortants made with the wt $\mathrm{B} / \mathrm{HN} / 76$ virus, the ca marker appears to arise from a combination of the genes of ca $B / A A / 1 / 66$, since no individual gene conferred this phenotype. However, with the wt $B /$ Texas/1/84 parent, as mentioned above, a single-gene NP reassortant did become cold-adapted in PCK cells. In addition to being tested in PCK instead of MDCK cells, one other possible reason for this inconsistency is that because the ca mutations enable growth, any wt gene which cannot function efficiently at $25^{\circ}$ will abrogate the appearance of the ca phenotype, obscuring any number of ca enabling genes. In our studies, all genes except the NS gene, showed at least one unique amino acid change in its predicted protein sequence. Thus, every ca viral gene which has been implicated in the direct expression of one or more of the three phenotypes, by the above genetic studies, was shown to contain nucleotide and amino acid changes from those of the wt gene. Recently, a non-ts revertant of the PA gene was isolated and sequenced, and the sequence reversion has been shown to involve the codon position, 1320, PA gene (Fig. 8). The experiments detailing this revertant gene are presented in the companion paper (Donabedian et al., 1988).

We have presented a catalog of changes that existed between the wt and $\mathrm{ca} B / \mathrm{AA} / 1 / 66$ viruses that should provide a valuable basis for understanding the mechanisms by which the ca donor virus confers the $c a, t s$, and att phenotypes to new ca vaccine reassortants. The changes that have occurred in the sequences of genes from phenotypic revertant viruses can be compared to those of the wt and ca B/AA/1/66 viruses in order to link specific mutations to specific phenotypes. This sequence information also provides a basis for the design of site mutagenesis experiments aimed at establishing directly the importance of some sequence sites to phenotypes not amenable to sin- gle-gene or revertant analysis (like the ca phenotype). Finally, understanding which of the six nonsurface genes of ca B/AA $1 / 66$ are essential for the stability and attenuation of the ca reassortant live virus vaccine, and which genes are not, can lead to the laboratory creation of more effective ca reassortant donor viruses.

\section{ACKNOWLEDGMENTS}

We thank Susan Donabedian for the expert technical help in the preparation and sequencing of viral RNA. The work in this paper was supported by Contract 1-Al-52564, National Institute of Allergy and Infectious Diseases, Development and Application Branch, Bethesda, Maryland 20892, and by a Biomedical Research Support Grant RR 05584-21.

\section{REFERENCES}

Akoto-Amanfu, E., Sivasubramanian, N., and Nayak, D. P. (1987). Primary structure of the polymerase acidic (PA) gene of an influenza $B$ virus (B/Sing/222/79). Virology 159, 147-153.

BRIEDIS. D. J., and LAMB, R. A. (1982). Influenza B virus genome: Sequences and structural organization of RNA segment 8 and the mRNAs coding for the $\mathrm{NS}_{1}$ and $\mathrm{NS}_{2}$ proteins. /. Virol. 42 , 186-193.

BRIEDIS, D. J., LAMB, R. A., and CHOPPIN, P. W. (1982). Sequence of RNA segment 7 of the influenza $B$ virus genome: Partial amino acid homology between the membrane proteins $\left(M_{1}\right)$ of influenza $A$ and $B$ viruses and conservation of a second open reading frame. Virology 116, 581-588.

Briedis, D. J., and TobIN, M. (1984). Influenza B virus genome: Complete sequence of the influenza $B / L$ ee $/ 40$ virus genome $R N A$ segment 5 encoding the nucleoprotein and comparison with the $\mathrm{B} /$ Singapore/222/79 nucleoprotein. Virology $133,448-455$.

Cox, N. J., Kitame, F., Klimov, A., Koennecke, I., and Kendal, A. P. (1986). Comparative studies of wild-type and cold-mutant (temperalure-sensilive) influeriza virus: Detection of mutations in all genes of the A/Ann Arbor/6/60(H2N2) mutant vaccine donor strain. Microbiol. Pathol. 1, 387-397.

DeBorde, D. C., Naeve, C. W., Herlocher, M. L., and MaAssab, H. F. (1986). Resolution of a common RNA sequencing ambiguity by terminal deoxynucleotidyl transterase. Anal. Biochem. 157, 275-282

DeBorde, D. C., Naeve, C. W., Herlocher, M. L., and MaAssab, H. F. (1987). Nucleotide sequences of the PA and PB1 genes of $B / A n n$ Arbor/1/66 virus: Comparison with genes of $B / L e e / 40$ and type A influenza viruses. Virus Res. 8, 33-41.

Donabedian, A. M., DeBorde, D. C., CoOK, S., Smitka, C. W., and MAASSAB, H. F. (1988). A mutation in the PA protein of coldadapted (ca) B/Ann Arbor/1/66 influenza virus associated with reversion of the ts phenotype and attenuated virulence. Virology $163,444-451$.

Donabedian, A. M., DeBorde, D. C., Cook, S., Smitka, C. W., and MAASSAB, H. F. (1988). A mutation in the PA protein of coldadapted (ca) B/Ann Arbor/1/66 influenza virus associated with reversion of the ts phenotype and attenuated virulence. Virology $163,444-451$.

Hiebert, S. W., Williams, M. A., and Lamb, R. A. (1986). Nucleotide seqeunce of RNA segment / of influenza B/Singapore/222/79: 
Maintenance of a second large open reading frame. Virology 155 , 747-751.

Keitel, W. A., CATE, T. R., and Couch, R. B. (1986). Evaluation of a cold-recombinant influenza $B$ vaccine. In "Options for the Control of Influenza" (A. P. Kendal and P. A. Patriarca, Eds.), pp. 287-291. A. R. Liss, New York.

Kemdirim, S., FALEFsky, J., and Briedis, D. J. (1986). Influenza B virus PB1 protein: Nucleotide sequence of the genome RNA segment predicts a high degree of structural homology with the corresponding influenza A virus polymerase protein. Virology 152, $126-135$.

Londo, D. R., Davis, A. R., and Nayak, D. P. (1983). Complete nucleotide sequence of the nucleoprotein gene of influenza $B$ virus. J. Virol. $47,642-648$.

MaAssab, H. F., DeBorde, D. C., Donabedian, A. M., and Smitka, C. W. (1985). Development of cold-adapted "master" strains for type B influenza virus vaccines. In "Vaccines 85 " (R. A. Lerner, R. M. Chanock, and F. Brown, Eds.), pp. 327-332. Cold Spring Harbor Laboratory, Cold Spring Harbor, NY.

MaAssab, H. F., Deborde, D. C., Donabedian, A. M., and Smitka, C. W. (1986). Prospects for influenza type B live attenuated vaccines. In "Cptions for the Control of Influenza" (A. P. Kendal and P. A. Patriarca, Eds.), pp. 271-286. A. R. Liss, New York.
MARTIN, R. (1987). Overcoming DNA sequencing artifacts: Stops and compressions. Focus 9, 8-10.

Mount, S. M. (1982). A catalog of splice junction sequences. Nucleic Acids Res. 10, 459-472.

PALESE P., and YOUNG, J. F. (1983). Molecular epidemiology of influenza virus. In "Genetics of Influenza Viruses" (P. Palese and D. W. Kingsbury, Eds.), pp. 321-336. Springer-Verlag, New York.

Parvin J. D., Moscona, A., Pan, W. T., Leider, J. M. and Palese, P. (1986). Measurement of the mutation rates of animal viruses: Influenza A virus and poliovirus type 1. J. Virol. 59, 377-383.

PEACOCK, A. C., and Dingman, C. W. (1968). Molecular weight estimation and separation of ribonucleic acid by electrophoresis in agarose-acrylamide composite gels. Biochemistry 7, 668-674.

PeATTIE, D. A. (1979). Direct chemical method for sequencing RNA. Proc. Natl. Acad. Sci. USA 76, 1760-1764.

QUEEN, C., and KORN, L. J. (1984). A comprehensive sequence analysis program for the IBM personal computer. Nucleic Acids Res. $12,581-599$.

Reeve, P., Pibermann, M., Bachmayer, H., Liehl, E., Moritz, A., Ganzinger, U., Hofmann, H., and Kunz, C. (1982). Studies in man with a cold-recombinant live influenza B virus vaccine. J. Med. Virol. 9, 1-9. 